\title{
A Game-theoretic Analysis of a Competitive Diffusion Process over Social Networks
}

\author{
Vasileios Tzoumas* $^{*}$ Christos Amanatidis ${ }^{\dagger} \quad$ Evangelos Markakis $^{\dagger}$
}

\begin{abstract}
We study a game-theoretic model for the diffusion of competing products in social networks. Particularly, we consider a simultaneous non-cooperative game between competing firms that try to target customers in a social network. This triggers a competitive diffusion process, and the goal of each firm is to maximize the eventual number of adoptions of its own product. We study issues of existence, computation and performance (social inefficiency) of pure strategy Nash equilibria in these games. We mainly focus on 2-player games, and we model the diffusion process using the known linear threshold model. Nonetheless, many of our results continue to hold under a more general framework for this process.

In more detail, we first exhibit that these games do not always possess pure strategy Nash equilibria, and we prove that deciding if an equilibrium exists is co-NP-hard. We then move on to investigate conditions for the existence of equilibria. We first illustrate why we cannot hope that games over networks with special in and out-degree distributions - e.g. power law — are more stable than others, concerning for example, the form of the improvement paths, or cycles that they induce. We then study necessary and sufficient conditions for the existence of pure Nash equilibria, both for the general case but for some special cases as well. Our conditions go through the existence of generalized ordinal potential functions. We also study the existence of $\epsilon$-generalized ordinal potentials (which yield $\epsilon$-approximate Nash equilibria) and provide tight upper bounds on the existence of such approximations. Finally, we study the Price of Anarchy and Stability for games with an arbitrary number of players. We conclude with a discussion of the effects on the payoff of a single player (or a coalition of players) as the number of players increases.
\end{abstract}

\footnotetext{
*National Technical University of Athens (NTUA), School of Electrical and Computer Engineering. Email: vdtzoumas@ gmail.com.

${ }^{\dagger}$ Athens University of Economics and Business (AUEB), Department of Informatics. Emails: \{amanatidis.c, markakis\}@ gmail.com.
} 


\section{Introduction}

Social networks have become a huge interdisciplinary research area with important links to various fields ranging from sociology and economics, to computer science, and mathematics. The growth of this area is also illustrated in recent books such as $[12,14,18]$.

A large part of research on social networks concerns the topic of diffusion of information (e.g., ideas, behaviors, trends). Mathematical models for diffusion processes have been proposed ever since $[16,29]$ and also later in [13]. Given such a model, some of the earlier works focused on the following optimization problem: find a set of nodes to target so as to maximize the spread of a given product (in the absence of any competitors). This problem was initially studied by Domingos and Richardson [11] and Kempe et al. [19] and subsequently by $[9,27]$. Their research builds on a "word-of-mouth" approach, where the initial adopters influence some of their friends, who in turn recommend it to others, and eventually a cascade of recommendations is created [22]. Within this framework, finding the most influential set of nodes is NP-hard, and approximation algorithms as well as heuristics have been developed for various models.

Different considerations, however, need to be made in the presence of multiple competing products in a market. In real networks, customers (i.e., nodes) end up choosing a product among several alternatives. Hence, one natural approach to model this competitive process is the use of game-theoretic analysis with the players being the firms that try to market their product. The game-theoretic approaches that have been proposed along this direction mainly split into two types. The first is to view the process as a Stackelberg game, where the competitors of a product first choose their strategy, and then a last mover needs to make a decision on the set of nodes to target $[6,7,8,20]$. This approach essentially reduces to the algorithmic question of finding the best response for the firm that moves last. The main results that have been obtained along this direction is that, in certain cases, the algorithm of [19], for the case of a single product, can be applied in the competitive environment as well. For more models and related problems under this context see also [4]. A different approach is to capture the competition as a simultaneous game, where firms pick their initial set of nodes at the same time, and then the diffusion process follows (after first taking care of ties). This was first proposed in [1], and has also been studied very recently by [15]. The approach of [1] and [15] as a standard noncooperative normal-form game is the focus of our work as well.

\subsection{Contributions}

Our work is an attempt to further understand game theoretic aspects of viral marketing. To this end, we first define in Section 2 a general framework for a competitive diffusion process in a social network, generalizing the model of [1]. This corresponds to a class of non-cooperative games where firms target customers in order to maximize the spread of their own product. We study issues of existence, computation, and performance (social inefficiency) of pure Nash equilibria (PNE). A key assumption in our work, adopted also by both $[1,15]$, is that we consider a one-stage game, i.e., all firms spend their budget in one step, a fact that renders natural the analysis of PNE. We use as instantiations of the competitive diffusion process the well-known linear threshold model, however some of our results also hold for more general local interaction schemes. In a nutshell, our main findings are that $i$ ) the graph structure on its own is not critical for determining, or characterizing the existence of PNE (formalized in Section 4.1); instead other factors, explained below, and in Section 4, in combination with the graph structure, play a key role in finding stable outcomes, and ii) PNE tend to be socially inefficient. Hence, competition among firms, in this context, may in the worst case lead to a poor outcome both for the firms, but also for the network users.

In more detail, we mostly deal with 2-player games, as in [15], and in Section 3 we first illustrate that such games may not possess PNE, even for simple graphs. On top of that, we also prove that it is co-NP-hard to decide whether a PNE exists for a given game. We then move on to investigate conditions for the existence of PNE. In Section 4, we begin with studying the improvement paths induced by our games. We exhibit that networks with special in and out-degree distributions - e.g. power law - are not expected to be more stable than others, in the sense that all possible dynamics can be realized essentially by any graph. Motivated by all these, we then focus on sufficient conditions for the existence of PNE via generalized ordinal potential functions. We also consider $\epsilon$-approximate generalized ordinal potentials, and we provide tight upper bounds on the existence of such approximations, as well as, polynomial time algorithms for computing approximate PNE. Finally, we study 
the Price of Anarchy and Stability for games with an arbitrary number of players, and we show that PNE (when they exist) can be quite inefficient. We conclude with a discussion of the effects on the payoff of a single player (or a coalition of players), as the number of competitors increases.

We view as one of the main contributions the fact that we unveil new decisive factors for the existence of PNE that are intertwined with structural characteristics of the underlying network. For example, some of the factors that play a role in our model for obtaining generalized ordinal potentials (exact, or approximate) involve $i$ ) the diffusion depth of a game (defined in Section 2 as the maximum possible duration of the diffusion process), ii) the ideal spread (defined as the maximum possible spread that a strategy can achieve) and iii) the diffusion collision factor (defined in Section 4.3 as a measure for comparing how two strategies of one player perform against a given strategy of another player). We advocate that our results motivate further empirical research on social networks for identifying a typical range of these quantities in real networks. Regarding the diffusion depth, some empirical research has already provided new insights for certain recommendation networks [22, 24].

\subsection{Related work}

Our work has been largely motivated by [1], (see also the corresponding errata [2, 31]). To the best of our knowledge, this was the first article to consider such simultaneous games over networks with the players being the firms. The diffusion process of [1] is a special case of our model, in particular, it is a linear threshold model where each firm is allowed to target only one node, and where the thresholds and the weights are equal to $1 /|N(v)|(N(v)$ being the neighbors of node $v)$. Also, their tie-breaking rule does not allow any nodes that face a dilemma to adopt any product. Our work considers the general class of linear threshold models, and in some cases our results hold even for arbitrary local interaction schemes beyond threshold models. In [1] the existence of pure Nash equilibria is linked to bounding the diameter of the graph. In our model we find that the diameter is not much correlated to existence. Instead we identify other parameters that influence the existence of equilibria.

Besides [1], a very recent related work is [15]. One of the major differences between [15] and our work is that they study the set of mixed Nash equilibria of a similar diffusion game, and focus on the Price of Anarchy, and another measure denoted as the Budget Multiplier. We, on the other hand, focus on pure Nash equilibria. Another difference is that [15] is studying stochastic processes whereas our local interaction schemes induce deterministic processes, as in [1]; nonetheless, in a context of a randomized diffusion process, our work can be interpreted as a worst-case analysis.

Other game-theoretic approaches have also been considered for social networks. One line of work concerns models of Stackelberg games as mentioned earlier [6, 7, 8, 20]. A different approach is to consider a game where the players are the individual nodes of the network, who have a utility function depending on their own choice, and that of their neighbors, see e.g., $[17,26,30]$. This leads to very different considerations.

\section{Preliminaries}

We define a game between two competing firms that simultaneously target subsets of consumers within a network, which in turn triggers a competitive diffusion process. Illustrative examples for some of the following definitions can be found in Appendix A.

\subsection{Social Networks}

The underlying structure of the social network is assumed static, and is modeled by a fixed finite directed graph $G=(V, E)$ with no parallel edges and no self-loops. Each node $v \in V$ represents an individual within the social network, while each directed edge $(u, v) \in E$ represents that $v$ can be influenced by $u$. As in [7, 15], we assume that there are two competing products (or trends, ideas, behavioral patterns) produced by two different firms $\mathcal{M}=\{1,2\}$. To each such product we assign a unique color. Throughout this work, we shall use the terms product, color, and firm interchangeably. Further, each node can adopt at most one product, and as with most of the literature, we assume that all adoptions are final; i.e., no node that has adopted a particular color will later alter its decision. Moreover, if a node has adopted a color, we shall refer to it as colored, or infected, and 
otherwise as white.

We denote the (in-)neighbors of a node $v$ as $N(v)=\{u \in V \mid(u, v) \in E\}$, i.e., $N(v)$ is the set of nodes that can influence $v$. Also, we denote as $d_{v}^{i n}$ and $d_{v}^{\text {out }}$ the in-degree and out-degree of $v$. The way that a node $v$ can be influenced by $N(v)$ is usually described by a local interaction scheme (LIS). Hence, a local interaction scheme is essentially a function that takes as input a node $v$, the status of its neighbors, a product $c$ under consideration, and possibly other characteristics of the graph, and determines if node $v$ is eligible to adopt this product. An example of a LIS, that was initially studied for the spread of a single product, is the linear threshold model (LTM) $[16,29]$. Under LTM, there is a weight $w_{u v} \in[0,1]$ for every edge $(u, v)$ such that for every node $v$, it holds that $\sum_{u \in N(v)} w_{u v} \leq 1$. Moreover, every node $v$ has a threshold value $\theta_{v} \in(0,1]$. The condition that needs to hold, under LTM, so that node $v$ can adopt a product $c$ is

$$
\sum_{u \in N(v)} \mathbb{I}_{u} w_{u v} \geq \theta_{v},
$$

where $\mathbb{I}_{u}$ is 1 if $u$ has already adopted product $c$, and zero otherwise. Another interaction scheme is the general threshold model, where the sum of weights is replaced by a function of the set of neighbors that have adopted the given product [19]. Note that in a local interaction scheme, the eligibility condition may hold for more than one product at a given time (e.g., under LTM this could happen if $\theta_{v}<1 / 2$ for some node $v$ ).

Given a local interaction scheme, and a set of competing firms, we consider the following competitive diffusion process, which evolves over discrete time steps:

The diffusion process. Initially each firm tries to infect a set of "seeds". The number of seeds for each firm may depend on its budget for advertising and marketing. We assume here that the firms have the same power so that in the beginning they can target a set of $k$ nodes each. Typically $k<<|V|$ but here we will not make any further assumption about how large $k$ can be.

- At time step $t=0$ : This is the initiation step. In the beginning, all nodes are colored white. If a node $v$ was targeted by a single firm $c$, then $v$ adopts product $c$. Since each firm may pick to target an arbitrary set of $k$ nodes, some overlaps may also occur. Thus, we assume that a tie-breaking criterion $\mathrm{TBC} 1$ is applied to resolve such dilemmas. This may be a global rule, or a rule that depends on each node.

- At time step $t>0$ : We look at each remaining white node and check if it is eligible to adopt any of the products, i.e., if the adoption condition, as determined by LIS, holds. For this, we take into account only the neighbors of $v$ that were infected up until time step $t-1$, hence the order with which we examine the white nodes does not matter. During this process, a white node $v$ may be eligible to adopt more than one product. To resolve such dilemmas a second tie-breaking criterion TBC2 should be considered, (it could be the same as $\mathrm{TBC} 1$ ). The process terminates at a time step $t$, when no white node is eligible to adopt any product. We allow that $\mathrm{TBC} 1$ may differ from $\mathrm{TBC} 2$, since $\mathrm{TBC} 2$ may depend on specific features of the diffusion process, whereas $\mathrm{TBC} 1$ occurs only at the initiation step.

A particular instance of a tie-breaking criterion, that we shall often use, is the rule that is also used in [17, 26], where ties are resolved in favor of the "best quality" product: all the individuals within the social network share a common reputation ordering, say $R^{\prec} \equiv 1 \succ 2$, over the products and in case of ties they decide according to $R^{\prec}$. We shall also see later that some of our results are independent of the tie-breaking rules.

Note. All definitions above can be generalized in a straightforward manner to an arbitrary number of $m$ firms, i.e., $\mathcal{M}=\{1, \ldots, m\}$. In Section 3 and Section 4 we focus mostly on the 2-player case. Section 5 deals with arbitrary $m$-player games as well.

Definition 1. A social network $\mathscr{N}$ is defined through the tuple (G, LIS, TBC1, TBC2).

\subsection{Strategic Games}

A game $\Gamma=(\mathscr{N}, \mathcal{M}, k)$ is induced by a social network $\mathscr{N}=(G$, LIS, TBC1, TBC2 $)$ and the set of firms $\mathcal{M}=\{1, \ldots, m\}$, which we shall refer to as a diffusion game. In a diffusion game, all participating firms 
choose simultaneously a set of $k$ seeds, which then triggers a diffusion process according to the interaction scheme and tie-breaking criteria of $\mathscr{N}$. We denote as $\mathcal{S}=\{S:|S|=k\}$ the set of available strategies, which is the same for each firm. We shall use the phrases strategy $S$ and subset $S$ interchangeably. A pure strategy profile is a vector $\mathbf{s}=\left(S_{1}, \ldots, S_{m}\right) \in \mathcal{S}^{m}$, where $S_{i}$ corresponds to the strategy played by player $i \in \mathcal{M}$. Also, we set $\mathbf{s}_{-\mathbf{i}} \equiv\left\{S_{1}, \ldots, S_{i-1}, S_{i+1}, \ldots, S_{m}\right\}$.

Given a strategy profile $\mathbf{s} \in \mathcal{S}^{m}$, the utility of firm $i \in \mathcal{M}$, denoted by $u_{i}(\mathbf{s})$, is the number of nodes that have been colored in $c_{i}$ by the end of the competitive diffusion process. Moreover, we denote the associated game matrix as $\Pi(\Gamma)$.

Additionally, a pure strategy profile $\mathbf{s} \in \mathcal{S}^{m}$ is a pure Nash equilibrium (PNE) of game $\Gamma$ if $u_{i}\left(S_{i}^{\prime}, \mathbf{s}_{-\mathbf{i}}\right) \leq u_{i}(\mathbf{s})$, $\forall i$ and $\forall S_{i}^{\prime}$.

An important parameter in our games is the so-called diffusion depth defined below.

Definition 2. The diffusion depth $D(\Gamma)$ of a game $\Gamma$ is defined as the maximum number of time steps that the competitive diffusion process may need, where the maximum is taken over all strategy profiles $\mathbf{s} \in \mathcal{S}^{m}$.

Observe that the diffusion depth can take values either lower, equal, or greater than the diameter of the underlying graph $G$.

Another important notion in our analysis is defined below. Consider a hypothetical scenario where only one player participates in the game. Then his payoff will not be obstructed by anybody else, and any strategy that he chooses achieves its best possible performance. This is useful for quantifying the players' utilities as we shall see later on.

Definition 3. Assume that only one player from $\mathcal{M}$ participates in the game, and let $S \in \mathcal{S}$ be one of his strategies. We define as ideal spread of $\mathbf{S}$, denoted by $H_{S}$, the set of nodes that have adopted by the end of the diffusion process the product of this player under strategy $S$. This includes the initial seed as well, i.e., $S \subseteq H_{S}$.

\section{Existence: Examples and Complexity}

We start with some remarks concerning the presentation. In Section 3 and Section 4 we consider mostly 2-player games. Furthermore, our results mainly hold for the linear threshold model but some of them can be generalized to arbitrary models. Whenever in stating a theorem, we do not specify a parameter of the network, it means that it holds independent of its value (e.g. in some results we do not specify the tie-breaking criteria, or the local interaction scheme).

The games that we study do not always possess PNE and we present an example below to illustrate this. We note that this is independent of the tie-breaking criteria used. For any other choice of such criteria (whether deterministic, or randomized, e.g., adopt randomly one of the products when both products are eligible) we can construct analogous examples.

Example 1. Consider the game $(\mathscr{N}, \mathcal{M}=\{1,2\}, k=1)$ over the graph of Figure 1 , where $\mathscr{N}=(G, \mathrm{LIS}=$ $\left.\mathrm{LTM}, \mathrm{TBC} 1=R^{\prec}, \mathrm{TBC} 2=R^{\prec}\right)$. It is easy to generalize this to a line with an arbitrary number of nodes. We assume that all the nodes have threshold 1 . Moreover, the edges are annotated with their corresponding weight with regard to $L T M$.

The game matrix is seen in Table 1 and it is easy to check that no PNE exists.

$$
n_{1} \stackrel{1}{\longrightarrow} n_{2} \stackrel{1}{\longrightarrow} n_{3}
$$

Figure 1: A network with underlying structure a line.

\begin{tabular}{c||c|c|c} 
& $n_{1}$ & $n_{2}$ & $n_{3}$ \\
\hline \hline$n_{1}$ & 3,0 & 1,2 & 2,1 \\
\hline$n_{2}$ & 2,1 & 2,0 & 1,1 \\
\hline$n_{3}$ & 1,2 & 1,1 & 1,0
\end{tabular}

Table 1: The payoff matrix for the game of Figure 1.

The example reveals that even simple graph structures may fail to have PNE. This holds for larger values of $k$ as well, and we have also found other examples with no PNE, where the graph $G$ is a cycle, a clique, or belongs to certain classes of trees (omitted from this version). 
For the sake of completeness, we present an additional example with $k>1$ and with nodes that have more than one neighbor, contrary to Example 1.

Example 2. Consider the 2-player game $(\mathscr{N}, \mathcal{M}=\{1,2\}, k=2)$ over the network in Figure 2, where again $\mathscr{N}=\left(G, \mathrm{LIS}=\mathrm{LTM}, \mathrm{TBC} 1=R^{\prec}, \mathrm{TBC} 2=R^{\prec}\right)$. The thresholds are all equal to 1 and the weights are seen in the figure. The game matrix in Table 2 reveals that no PNE exists.

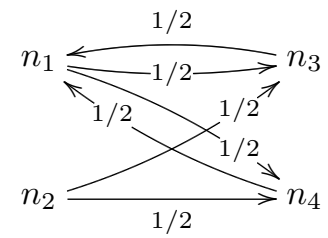

\begin{tabular}{c||c|c|c|c|c|c} 
& $n_{1} n_{2}$ & $n_{1} n_{3}$ & $n_{1} n_{4}$ & $n_{2} n_{3}$ & $n_{2} n_{4}$ & $n_{3} n_{4}$ \\
\hline \hline$n_{1} n_{2}$ & 4,0 & 3,1 & 3,1 & 3,1 & 3,1 & 2,2 \\
\hline$n_{1} n_{3}$ & 2,1 & 2,0 & 2,1 & 2,1 & 2,2 & 2,1 \\
\hline$n_{1} n_{4}$ & 2,1 & 2,1 & 2,0 & 2,2 & 2,1 & 2,1 \\
\hline$n_{2} n_{3}$ & 2,1 & 2,1 & 2,2 & 2,0 & 2,1 & 2,1 \\
\hline$n_{2} n_{4}$ & 2,1 & 2,2 & 2,1 & 2,1 & 2,0 & 2,1 \\
\hline$n_{3} n_{4}$ & 2,2 & 2,1 & 2,1 & 3,1 & 3,1 & 3,0
\end{tabular}

Figure 2: A network with underlying structure an equitable graph.

Table 2: The payoff matrix for the game of Figure 2.

Given these examples, the next natural question is whether it is easy to decide if a given game has at least one PNE. We assume that the input to this algorithmic question is not the game matrix, which can be exponentially large, but simply the graph $G$ and a description of the local interaction scheme, e.g., for LTM, the threshold and the weights suffice. Note that for $k=O(1)$ the problem is easy, hence the computational challenge is for larger values of $k$. We establish the following hardness result.

Theorem 1. Deciding whether a game $\left(\left(G, \mathrm{LIS}=\mathrm{LTM}, \mathrm{TBC} 1=R^{\prec}, \mathrm{TBC} 2=R^{\prec}\right), \mathcal{M}, k\right)$ has a PNE is co-NP-hard and belongs to $\Sigma_{2}^{p}$.

Remark 1. The reduction in the proof of Theorem 1 produces instances where the network is a directed acyclic graph (DAG) and the diffusion depth is $D=3$.

We shall see later that DAGs also play a role on identifying conditions for the existence of PNE (Section 4). The proof of Theorem 1 is given in Appendix B and is based on a reduction from 3SAT. We remark that the instances of the reduction are not weakly connected graphs but we can adjust our reduction to produce such families too.

Note that we have not obtained membership in the class co-NP. This is because there seems to be no short certificate for checking that a game does not have any PNE (one would need to check all strategy profiles). It is an open problem to determine if the problem is complete for $\Sigma_{2}^{p}$. Another open problem would be to determine the complexity for games with diffusion depth $D=1$, or $D=2$.

\section{Towards Characterizations}

To understand better the issue of existence of PNE, we start with quantifying the utility functions $u_{i}: \mathcal{S}^{2} \mapsto \mathbb{N}$, $i \in \mathcal{M}=\{1,2\}$. For this we need to introduce some important notions. A convenient way to calculate the utility of a player under a profile $\mathbf{s}$, is by utilizing the definition of $H_{S}$ in Section 2, which is the ideal spread of a product if the firm was playing on its own and used $S$ as a seed. In the presence of a competitor, the firm will lose some of the nodes that belong to $H_{S}$. The losses happen due to three reasons. First, the competitor may have managed to infect a node at an earlier time step than the step that the firm would reach that node. Second, the firm may lose nodes due to the tie-breaking criteria, if both firms are eligible to infect a node at the same time step. Finally, there may be nodes that belong to $H_{S}$, but the firm did not manage to infect enough of their neighbors so as to color them as well. These nodes either remain white, or are eventually infected by the other player. All these are captured below:

Definition 4. Consider a game $((G, L I S, T B C 1, T B C 2), \mathcal{M}, k)$, and a strategy profile $\mathbf{s}=\left(S_{1}, S_{2}\right)$. For $i \in$ $\{1,2\}$,

$i$. we denote by $\alpha_{i}(\mathbf{s})$ the number of nodes that belong to $H_{S_{i}}$, and under profile $\mathbf{s}$, player $i$ would be eligible to color them at some time step $t$ but the other player has already infected them at some earlier time step $t^{\prime}<t$ (e.g., this may occur under the threshold model when $\theta_{v}<1 / 2$ for some node $v$ ). 
ii. we denote by $\beta_{i}(\mathbf{s})$ the number of nodes in $H_{S_{i}}$, such that under profile $\mathbf{s}$, both firms become eligible to infect them at the same time step, and due to tie-breaking rules, they get infected by the competitor of $i$.

iii. we denote by $\gamma_{i}(\mathbf{s})$ the number of nodes that belong to $H_{S_{i}}$, but under $\mathbf{s}$, firm i never becomes eligible to infect them (because $i$ did not manage to color the right neighbors under $\mathbf{s}$ ).

Finally, we set $\alpha_{i, \max }$ (respectively $\beta_{i, \max }, \gamma_{i, \max }$ ) to be the maximum value of $\alpha_{i}(\mathbf{s})$ over all valid strategy profiles and also $\alpha_{\max }=\max \left\{\alpha_{1, \max }, \alpha_{2, \max }\right\}$ (similarly for $\beta_{\max }$, and $\gamma_{\max }$ ). We refer the reader to the example in Appendix A for an illustration of these concepts.

When we use $R^{\prec}$ for tie-breaking, clearly $\beta_{1}(\mathbf{s})=0$, hence the following is straightforward.

Lemma 1. Consider the 2-player game $\left(\left(G, L I S, T B C 1=R^{\prec}, T B C 2=R^{\prec}\right), \mathcal{M}, k\right)$. The utility functions of the players, given a strategy profile $\mathbf{s}=\left(S_{1}, S_{2}\right) \in \mathcal{S}^{2}$, are

$$
\begin{aligned}
& u_{1}(\mathbf{s})=\left|H_{S_{1}}\right|-\alpha_{1}(\mathbf{s})-\gamma_{1}(\mathbf{s}), \\
& u_{2}(\mathbf{s})=\left|H_{S_{2}}\right|-\alpha_{2}(\mathbf{s})-\beta_{2}(\mathbf{s})-\gamma_{2}(\mathbf{s}) .
\end{aligned}
$$

\subsection{Realizability of Improvement Paths}

Following Section 3, we unwind further the richness and complexity of our games motivated by the study of their improvement paths. We establish that one of the main structural properties of social networks, their degree distribution, does not play a role on its own to the existence, and structure of equilibria. This fact motivates the search for other important parameters related to the existence of exact, or approximate PNE, which is the topic of the next subsections within Section 4.

An improvement path is any sequence $\left(\mathbf{x}_{1}, \mathbf{x}_{2}, \ldots, \mathbf{x}_{j}, \ldots\right)$ of strategy profiles $\mathbf{x}_{j} \in \mathcal{S}^{2}$ such that for every $j$ the strategy profiles $\mathbf{x}_{j}$ and $\mathbf{x}_{j+1}$ differ in exactly one coordinate, say the $i(j)$-th, i.e., only player $i(j)$ has switched to another strategy, and also $u_{i(j)}\left(\mathbf{x}_{j}\right)<u_{i(j)}\left(\mathbf{x}_{j+1}\right), \forall j \geq 1$. It is called a best response path if also $u_{i(j)}\left(\mathbf{x}_{j+1}\right)=\max _{x \in \mathcal{S}} u_{i(j)}\left(x,\left(\mathbf{x}_{j+1}\right)_{-i(j)}\right)$. Furthermore, a finite path $\left(\mathbf{x}_{1}, \ldots, \mathbf{x}_{n}\right)$, is called an improvement cycle if it is an improvement path, $\mathbf{x}_{1}=\mathbf{x}_{n}$, and $\mathbf{x}_{j} \neq \mathbf{x}_{j+1}$, for every $j \in\{1, \ldots, n-1\}$.

A well-known sufficient condition for existence of PNE is the Finite Improvement Property (FIP), saying that all improvement paths are finite [25] (e.g., consider congestion games). In our case, the FIP does not hold, but in order to find conditions for the existence of PNE, one could still try to understand how do cycles occur. For example, do the cycles have some particular form? Does the degree distribution affect the formation of cycles? We obtain a negative result in this direction, showing that essentially in any given graph, any possible set of cycles may be realized, independent of its structure.

We proceed with some more definitions. Given a finite 2-player game $\Gamma$, played on a $r \times r$ matrix, let $P(\Gamma)$ denote the set of all improvement paths (including infinite ones) that are induced by the game starting from any entry in the matrix. Let $P$ denote any possible set of consistent improvement paths (including cycles) that can be created on a $r \times r$ matrix. By a consistent set we mean that if, e.g., there is a path with a move from entry $(i, j)$ of the matrix to $(i, l)$, then there cannot be another path in $P$ that contains a move from $(i, l)$ to $(i, j)$. We say that $P$ is realizable if there is a game $\Gamma$ such that $P=P(\Gamma)$. We show that any such set $P$ is realizable by the family of our games, essentially by any graph. Hence all possible dynamics can be captured by these games.

To prove our claim, we will argue about an appropriate submatrix of the games we construct, since some strategy profiles may need to be eliminated due to domination. Particularly, we need the following form of domination.

Definition 5. Given a 2-player game, assume that $\mathcal{S}=X \cup Y$, where $X \cap Y=\emptyset$. We say that $X$ is a sink in $\mathcal{S}$, if at least one of the following holds:

$$
\begin{aligned}
\text { i. } & \forall(a, b) \in Y \times(Y \cup X), \exists x \in X \text { such that } u_{1}(x, b)>u_{1}(a, b) \text {, and } \forall(a, b) \in X \times Y, \exists x \in X \text { such that } \\
& u_{2}(a, x)>u_{2}(a, b) . \\
\text { ii. } & \forall(a, b) \in(Y \cup X) \times Y, \exists y \in X \text { such that } u_{2}(a, y)>u_{2}(a, b) \text {, and } \forall(a, b) \in Y \times X, \exists x \in X \text { such that } \\
& u_{1}(x, b)>u_{1}(a, b) .
\end{aligned}
$$


Essentially, the definition says that any improvement path that is not a cycle (generally, any best response path), starting from the $Y$-region of the matrix, will eventually come to the $X$-region.

Given a 2-player game, we let $\mathcal{S}_{\mathcal{D}}$ denote a minimal sink in $\mathcal{S}$, and $\Pi\left(\mathcal{S}_{\mathcal{D}}, \mathcal{S}_{\mathcal{D}}\right)$ be the restriction of the game matrix over this set of strategies. We can now state the following theorem, which proof is given in Appendix C.

Theorem 2. For any $r \geq 2$, any consistent set of improvement paths (including cycles) formed on a $r \times r$ matrix, is realizable by an appropriately chosen game on a $D A G\left(\left(G, L I S=\operatorname{LTM}\left(w_{u v} \geq \theta_{v}, \forall(u, v) \in E\right), T B C 1=\right.\right.$ $\left.\left.R^{\prec}, T B C 2=R^{\prec}\right), \mathcal{M}, k\right)$, on the restriction to a submatrix $\Pi\left(\mathcal{S}_{\mathcal{D}}, \mathcal{S}_{\mathcal{D}}\right)$, where $\mathcal{S}_{\mathcal{D}}$ is a minimal sink with $\left|\mathcal{S}_{\mathcal{D}}\right|=r$.

Finally, we have also shown that the same result holds not only for games on graphs that are DAGs. Essentially all graph structures can be used so as to produce families of games that realize all dynamics. Given a graph $G$, let $P_{i n}$ and $P_{\text {out }}$ be the in and out-degree distributions of $G$, i.e., $P_{i n}(i)$ is the number of nodes with in-degree equal to $i$. The following can be proved using Theorem 2 .

Corollary 1. Consider a graph $G^{\prime}=(V, E)$ with in and out-degree distributions, $P_{i n}$, and $P_{\text {out }}$. There exists a class of games $\left(\left(G \in \mathcal{F}, L I S=L T M\left(w_{u v} \geq \theta_{v}, \forall(u, v) \in E\right), T B C 1=R^{\prec}, T B C 2=R^{\prec}\right), \mathcal{M}, k\right)$, where $\mathcal{F}$ is a family of graphs with the same set of nodes $V$, such that:

i. each $G \in \mathcal{F}$ has degree distributions $P_{\text {in }}^{G}, P_{\text {out }}^{G}$ such that for all $i,\left|P_{\text {in }}(i)-P_{\text {in }}^{G}(i)\right| /|V| \rightarrow 0$, as $|V| \rightarrow \infty$, and the same holds for $P_{\text {out }}$ and $P_{\text {out }}^{G}$.

ii. All sets of consistent improvement paths are realizable over the games played on $\mathcal{F}$ in $\Pi\left(\mathcal{S}_{\mathcal{D}}, \mathcal{S}_{\mathcal{D}}\right)$, where $\mathcal{S}_{\mathcal{D}}($ for each $G \in \mathcal{F})$ is as in Theorem 2, and $r \geq 3$.

Its proof is given in Appendix $\mathrm{C}$.

This result discloses the richness of our games, but above all it severely mitigates the role of the widely studied degree distribution of networks $[22,5,28]$ to the stability of the involved games. Hence, we advocate, in the following, that one needs to take into account the effects of other properties as well, such as the diffusion depth, the ideal spread, and the diffusion collision factor; these properties directly interact with the underlying network structure and influence the diffusion, and the general game dynamics.

\subsection{Conditions for the Existence of a PNE}

In this subsection, we formulate first two conditions that need to hold at any PNE, and then we use the notion of ordinal potentials to argue about existence of PNE. The results in this and the next subsection shall enhance our intuition over the games under consideration, and disclose further their stability properties.

Theorem 3. Given a game $\left(\left(G, L I S, T B C 1=R^{\prec}, T B C 2=R^{\prec}\right), \mathcal{M}, k\right)$, if the strategy profile $\mathbf{s}=\left(S_{1}, S_{2}\right) \in$ $\mathcal{S}^{2}$ is a PNE, then it holds that $\left|H_{S_{1}}\right|+\left|H_{S_{2}}\right| \geq\left|H_{\max }\right|-\gamma_{1}\left(S_{\max }, S_{2}\right)$, where $\left|H_{\max }\right|=\max _{S \in \mathcal{S}}\left\{\left|H_{S}\right|\right\}$, and $S_{\text {max }}=\operatorname{argmax}_{S \in \mathcal{S}}\left\{\left|H_{S}\right|\right\}$.

Its proof is given in Appendix D. The intuition behind Theorem 3 is that strategies that do not have (collectively) large ideal spread cannot form a PNE. We now derive a different necessary condition. We say that a set $X \subseteq V$ is reachable from a strategy $S \in \mathcal{S}$ if and only if $X \subseteq H_{S}$.

Theorem 4. Given a game $\left(\left(G, L I S, T B C 1=R^{\prec}, T B C 2\right), \mathcal{M}, k\right)$, if the strategy profile $\mathbf{s}=\left(S_{1}, S_{2}\right) \in \mathcal{S}^{2}$ is a PNE, then $S_{1}$ is not reachable from $S_{2}$.

Its proof is given in Appendix D. Moreover, observe that in Theorem $4 \mathrm{TBC} 2$ is arbitrary.

Corollary 2. A game $\left(\left(G, L I S, T B C 1=R^{\prec}, T B C 2\right), \mathcal{M}, k\right)$ in which the strategies in $\mathcal{S}$ are all reachable one from the other has no PNE.

An interesting consequence of Corollary 2 is that the family of games $\left(\left(G, \operatorname{LIS}=\operatorname{LTM}\left(w_{u v} \geq \theta_{v}, \forall(u, v) \in\right.\right.\right.$ $\left.E), \operatorname{TBC} 1=R^{\prec}, \mathrm{TBC} 2, \mathcal{M}=\{1,2\}, k\right)$, where $G$ is a simple cycle does not have PNE (independent of what $\mathrm{TBC} 2$ is). 
We now move on to generalized ordinal potentials. A function $P: \mathcal{S}^{2} \mapsto \mathbb{R}$ is a generalized ordinal potential [25] (GOP) for a game $\Gamma$ if $\forall i \in \mathcal{M}, \forall \mathbf{s}_{-\mathbf{i}} \in \mathcal{S}^{m-1}$, and $\forall x, z \in \mathcal{S}$,

$$
u_{i}\left(x, \mathbf{s}_{-\mathbf{i}}\right)>u_{i}\left(z, \mathbf{s}_{-\mathbf{i}}\right) \Rightarrow P\left(x, \mathbf{s}_{-\mathbf{i}}\right)>P\left(z, \mathbf{s}_{-\mathbf{i}}\right) .
$$

If $\Gamma$ admits a GOP and is also finite (as our games are), the FIP property holds (see Section 4.1), and all improvement paths terminate at a PNE $[25,32]$. On the other hand, in our games the existence of PNE is not equivalent with the FIP property; we can construct games that possess PNE, but do not admit a GOP (see Example 3 below). Instead, we continue with a set of necessary conditions on the existence of a GOP.

Example 3. Consider the game $(\mathscr{N}, \mathcal{M}=\{1,2\}, k=1)$ over the social network of Figure 3, where $\mathscr{N}=$ $\left(G, \mathrm{LIS}=\mathrm{LTM}, \mathrm{TBC} 1=R^{\prec}, \mathrm{TBC} 2=R^{\prec}\right)$. We assume that all the nodes have threshold 1 , except of node $n_{9}$ that has $1 / 2$. Moreover, the edges $\left(n_{i}, n_{j}\right)$, where $i, j \in\{1, \ldots, 9\}$, are annotated with their corresponding weight with regard to the LTM.

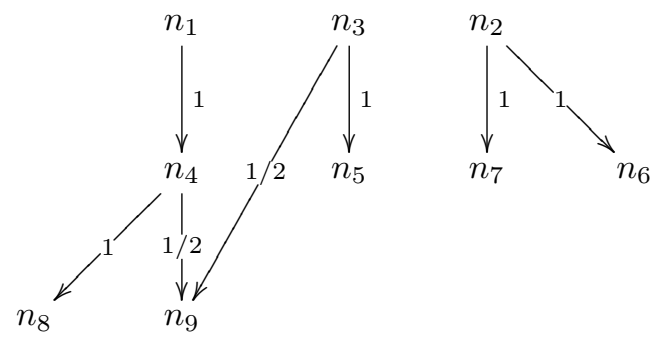

Figure 3: The network of Example 3.

The involved game matrix is as in Table 3. It can be verified that the strategy profile $\left(n_{1}, n_{2}\right)$ constitutes a PNE. On the other hand, the following improvement cycle exists: $\left(n_{1}, n_{1}\right) \rightarrow\left(n_{1}, n_{4}\right) \rightarrow\left(n_{2}, n_{4}\right) \rightarrow\left(n_{2}, n_{1}\right) \rightarrow$ $\left(n_{1}, n_{1}\right)$. Note that this path is also a best response path.

\begin{tabular}{c||c|c|c|c|c|c|c|c|c} 
& $n_{1}$ & $n_{2}$ & $n_{3}$ & $n_{4}$ & $n_{5}$ & $n_{6}$ & $n_{7}$ & $n_{8}$ & $n_{9}$ \\
\hline \hline$n_{1}$ & 4,0 & 4,3 & 3,3 & 1,3 & 4,1 & 4,1 & 4,1 & 3,1 & 3,1 \\
\hline$n_{2}$ & 3,4 & 3,0 & 3,3 & 3,3 & 3,1 & 2,1 & 2,1 & 3,1 & 3,1 \\
\hline$n_{3}$ & 3,3 & 3,3 & 3,0 & 3,2 & 2,1 & 3,1 & 3,1 & 3,1 & 2,1 \\
\hline$n_{4}$ & 3,1 & 3,3 & 3,2 & 3,0 & 3,1 & 3,1 & 3,1 & 2,1 & 2,1 \\
\hline$n_{5}$ & 1,4 & 1,3 & 1,2 & 1,3 & 1,0 & 1,1 & 1,1 & 1,1 & 1,1 \\
\hline$n_{6}$ & 1,4 & 1,2 & 1,3 & 1,3 & 1,1 & 1,0 & 1,1 & 1,1 & 1,1 \\
\hline$n_{7}$ & 1,4 & 1,2 & 1,3 & 1,3 & 1,1 & 1,1 & 1,0 & 1,1 & 1,1 \\
\hline$n_{8}$ & 1,3 & 1,3 & 1,3 & 1,2 & 1,1 & 1,1 & 1,1 & 1,0 & 1,1 \\
\hline$n_{9}$ & 1,3 & 1,3 & 1,2 & 1,2 & 1,1 & 1,1 & 1,1 & 1,1 & 1,0
\end{tabular}

Table 3: The payoff matrix for the game of Figure 3.

Lemma 2. The game $\left(\left(G, L I S, T B C 1=R^{\prec}, T B C 2\right), \mathcal{M}, k\right)$ cannot admit a generalized ordinal potential if

i. $\exists\left(S_{1}, S_{2}\right) \in \mathcal{S}^{2}, S_{1} \neq S_{2}$, such that $S_{1}$ is reachable from $S_{2}$, and $S_{2}$ is reachable from $S_{1}$.

ii. $\exists\left(S_{1}, S_{2}\right) \in \mathcal{S}^{2}, S_{1} \neq S_{2}$, such that $\left|H_{S_{1}}\right|=\left|H_{S_{2}}\right|$, and $S_{1}$ is reachable from $S_{2}$, or $S_{2}$ is reachable from $S_{1}$.

iii. $\exists\left(S_{1}, S_{2}\right) \in \mathcal{S}^{2}, S_{1} \neq S_{2}$, such that $\left|H_{S_{1}}\right|=\left|H_{S_{2}}\right|$.

iv. $\exists\left(S_{1}, S_{2}\right) \in \mathcal{S}^{2}, S_{1} \neq S_{2}$, such that $\left|H_{S_{1}}\right|>\left|H_{S_{2}}\right|$, and $u_{1}\left(S_{1}, S_{2}\right)<\left|H_{S_{2}}\right|$. 
The Lemma suggests that many classes of our games may not admit a GOP — in the next subsection we shall approximate how close to admitting a GOP these games are. The fact is elucidated further through the following corollary, where we assume $k=1$, i.e., each player has to pick a single node, therefore, the only reasonable strategies are the nodes $u$ for which there exists at least one edge $(u, v)$ such that $w_{u v} \geq \theta_{v}$.

Corollary 3. If the game $\left(\left(G, L I S=L T M, T B C 1=R^{\prec}, T B C 2=R^{\prec}\right), \mathcal{M}, k=1\right)$ admits a generalized ordinal potential, then

i. G contains a DAG that includes the set $\left\{u \mid \exists v \in V\right.$, such that $\left.w_{u v} \geq \theta_{v}\right\}$.

ii. if $w_{u v} \geq \theta_{v}$ for every edge $(u, v) \in E$, then $G$ has to be a $D A G$.

Corollary 3 shows that for the case of $k=1$, the conditions on the ideal spreads implied by Lemma 2, enforce the graph to have the special structure of a DAG. But clearly not all DAGs admit a GOP as has been demonstrated in Example 1.

We now show that arguing about games $\left(\left(G, \mathrm{LIS}=\mathrm{LTM}, \mathrm{TBC} 1=R^{\prec}, \mathrm{TBC} 2=R^{\prec}\right), \mathcal{M}=\{1,2\}, k\right)$ with $k>1$ can be reduced to arguing about games with $k=1$. Specifically, consider a game with $k>1$ over a graph $G$ : We define a graph $G_{s}=\left(V_{s}, E_{s}\right)$ such that

1. $V_{s} \equiv V \cup X$, where $|X|=\left(\begin{array}{c}|V| \\ k\end{array}\right)$. Further, we associate each node $x \in X$ with a single strategy $S_{x} \in \mathcal{S}$.

2. $E_{c} \equiv E \cup E_{X}$, where $E_{X} \equiv\left\{(x, v) \mid x \in X, v \in S_{x}\right\}$, and $\forall(x, v) \in E_{X}, w_{x v} \equiv \theta_{v}$, with regard to the LTM.

Thus, when the players in the game with $k=1$ over the graph $G_{s}$ initiate the nodes $x_{1}, x_{2} \in X$, they infect at the first step of the competitive diffusion process the same subset of nodes as they would infect at the initiation step of the original game (over graph $G$, and with $k>1$ ) if they were playing the strategies $S_{x_{1}}$, and $S_{x_{2}}$. Therefore, we have the following lemma.

Lemma 3. Given a game $\left(\left(G, L I S=L T M, T B C 1=R^{\prec}, T B C 2=R^{\prec}\right), \mathcal{M}=\{1,2\}, k>1\right)$, the corresponding game on $G_{s}$ with $k=1$ has diffusion depth $D+1$, and induces a game matrix $\Pi_{s}$, and a subset of strategies $X$ such that the restriction $\Pi_{s}(X, X)$ on $X$ has the same set of PNE as the original game.

We note that the construction of graph $G_{s}$ is done in exponential time. Thereby, although it is useful for arguing about the existence of PNE, it may not be used for actually computing them.

We finally derive a sufficient condition for the existence of a GOP.

Theorem 5. Consider a game $\left(\left(G, L I S, T B C 1=R^{\prec}, T B C 2\right), \mathcal{M}, k\right)$, and suppose that we order the set of the available strategies so that $\left|H_{S_{1}}\right| \geq \ldots \geq\left|H_{S_{|\mathcal{S}|}}\right|$. If for all $i \in\{1, \ldots,|\mathcal{S}|-1\}$ it is

$$
\left|H_{S_{i+1}}\right| \leq\left\lfloor\frac{\left|H_{S_{i}}\right|+\max \left\{\gamma_{1}\left(S_{i}, S_{i+1}\right), \gamma_{2}\left(S_{i}, S_{i+1}\right)\right\}}{2}\right\rfloor
$$

then the game admits a generalized ordinal potential. Moreover, all its PNE have the form $\left(S_{\max }, S_{2}\right)$, where $S_{\max } \equiv \operatorname{argmax}_{S \in \mathcal{S}}\left\{\left|H_{S}\right|\right\}$.

The proof is in Appendix D. For an interpretation of Theorem 5, suppose that we consider the max term to be zero in (3). Then, a GOP exists if all ideal spreads are well separated, and Player 2 can never hope to take more than half of the nodes that Player 1 would get ideally (see the introductory example for this theorem, in Appendix A).

The condition of Theorem 5 can be relaxed so that not all ideal spreads need to be well separated, e.g., in certain cases where there is no overlap between the ideal spreads of some strategies. For example, when $G$ is a full and complete $d$-ary tree, $d \geq 2$, then (3) does not hold but using similar arguments as in the proof of Theorem 5 we have:

Corollary 4. The games of the form $\left(\left(G, \operatorname{LIS}=\operatorname{LTM}\left(w_{u v} \geq \theta_{v}, \forall(u, v) \in E\right), T B C 1=R^{\prec}, T B C 2=\right.\right.$ $\left.\left.R^{\prec}\right), \mathcal{M}, k=1\right)$, where $G$ is a full and complete d-ary tree, admit a GOP. 


\subsection{Quantifying Instability}

The previous sections on existence and complexity motivate our next discussion on approximate PNE. Overall, the main conclusion of this subsection is that even though PNE do not always exist, we do have in certain cases approximate equilibria with a good quality of approximation, and we can also compute them in polynomial time.

A strategy profile $\mathbf{s}$ is an $\epsilon$-PNE, if no agent can benefit more than $\epsilon$ by unilaterally deviating to a different strategy, i.e., for every $i \in \mathcal{M}$, and $S_{i}^{\prime} \in \mathcal{S}$ it holds that $u_{i}\left(S_{i}^{\prime}, \mathbf{s}_{-\mathbf{i}}\right) \leq u_{i}(\mathbf{s})+\epsilon$. Recall that in our case, utilities are measured by the number of nodes infected by each player, hence all utilities are integers in $\{0, \ldots,|V|\}$, and $\epsilon$ also takes integer values ${ }^{1}$. Additionally, a function $P: \mathcal{S}^{2} \mapsto \mathbb{R}$ is an $\epsilon$-generalized ordinal potential ( $\epsilon$-GOP) for a game $\Gamma$ (see [10]) if $\forall i \in \mathcal{M}, \forall \mathbf{s}_{-\mathbf{i}} \in \mathcal{S}^{2}, \forall x, z \in \mathcal{S}, u_{i}\left(x, \mathbf{s}_{-\mathbf{i}}\right)>u_{i}\left(z, \mathbf{s}_{-\mathbf{i}}\right)+\epsilon \Rightarrow P\left(x, \mathbf{s}_{-\mathbf{i}}\right)>P\left(z, \mathbf{s}_{-\mathbf{i}}\right)$. Such a function $P$ yields directly the existence of $\epsilon$-PNE. We first obtain such a potential function for games that have diffusion depth $D=1$, based on the ideal spread of the players' strategies and on the quantification of the utility functions in the beginning of Section 4 (Definition 4).

Theorem 6. Any game $\Gamma=\left(\left(G, L I S, T B C 1=R^{\prec}, T B C 2=R^{\prec}\right), \mathcal{M}, k\right)$, where $D(\Gamma)=1$, admits the function $P(\mathbf{s})=\left(1+\beta_{\max }+\gamma_{\max }\right)\left|H_{S_{1}}\right|+\left|H_{S_{2}}\right|-\beta_{2}(\mathbf{s})-\gamma_{2}(\mathbf{s})$, as a $k$-GOP. Moreover, a $k$-PNE can be computed in polynomial time.

The last assertion of Theorem 6 is easy to see since the value of the function $P(\mathbf{s})$ is at most $O\left(|V|^{2}\right)$. Therefore, by following an improvement path (with improvements of more than $k$ ), we can find an approximate PNE quite efficiently.

Note that this holds for any local interaction scheme, and not just the linear threshold model. Theorem 6 implies that when $D(\Gamma)=1$ and $k$ is small, we can have a good quality of approximation. E.g., for $k=O(1)$, or $O(\log |V|)$ (or generally for $k=o(|V|)$ ), and as $|V| \rightarrow \infty$, we can have approximate equilibria where any node can additionally gain only a negligible fraction of the graph by deviating (i.e., under the normalization of utilities it would be $\epsilon \rightarrow 0$ as $|V|$ becomes large).

For games with higher diffusion depth, we define below an important parameter that captures the quality of approximation we can achieve in worst case via $\epsilon$-GOP.

Definition 6. $i$. Given a 2-player game, and two strategy profiles $\mathbf{s}=\left(S_{1}, S_{2}\right), \mathbf{s}^{\prime}=\left(S_{1}^{\prime}, S_{2}\right)$, the diffusion collision factor of player 1 for strategy $S_{1}^{\prime}$ compared to $S_{1}$, given $S_{2}$, is defined as $D C_{1}\left(S_{1}^{\prime}, S_{1} \mid S_{2}\right) \equiv$ $\left(\alpha_{1}\left(\mathbf{s}^{\prime}\right)+\gamma_{1}\left(\mathbf{s}^{\prime}\right)\right)-\left(\alpha_{1}(\mathbf{s})+\gamma_{1}(\mathbf{s})\right)$.

ii. Similarly, for $\mathbf{s}=\left(S_{1}, S_{2}\right), \mathbf{s}^{\prime}=\left(S_{1}, S_{2}^{\prime}\right)$, the diffusion collision factor of Player 2 for $S_{2}^{\prime}$ compared to $S_{2}$, given $S_{1}$, is defined as $D C_{2}\left(S_{2}^{\prime}, S_{2} \mid S_{1}\right) \equiv\left(\alpha_{2}\left(\mathbf{s}^{\prime}\right)+\gamma_{2}\left(\mathbf{s}^{\prime}\right)\right)-\left(\alpha_{2}(\mathbf{s})+\gamma_{2}(\mathbf{s})\right)$.

In order to understand this new notion, recall from Equation (1) that, given a profile $\mathbf{s}, \alpha_{1}(\mathbf{s})+\gamma_{1}(\mathbf{s})$ denotes the number of nodes that Player 1 does not infect due to the presence of Player 2 in the market; this fact directly yields some intuition for the definition of $D C_{1}$. This is not exactly the case for $D C_{2}$, as the $\beta_{2}$-term is missing (see Eq. (2)); nonetheless, it turns out that it suffices to define $D C_{2}$ in a uniform manner as $D C_{1}$, when using $R^{\prec}$ for ties. Finally, we set $D C_{\max }$ to be the maximum possible diffusion collision factor.

Theorem 7. Any game $\Gamma=\left(\left(G, L I S, T B C 1=R^{\prec}, T B C 2=R^{\prec}\right), \mathcal{M}, k\right)$, where $D(\Gamma) \geq 2$, admits the function $P(\mathbf{s})=x_{1}\left|H_{S_{1}}\right|+\left|H_{S_{2}}\right|-\beta_{2}(\mathbf{s})$, as a $D C_{\max }-G O P$, where $x_{1}$ is any number satisfying $x_{1}>\beta_{\max }$. Moreover, a $D C_{\text {max }}-P N E$ can be computed in polynomial time.

The proofs of Theorems 6,7 are given in Appendix E. The approximations of $k$ and $D C_{\max }$ are tight for LIS=LTM, and we provide the corresponding examples in the same Appendix.

\subsection{The Special Case $D(\Gamma)=k=1$}

We finish Section 4 with a special case that has received some interest in empirical works. Some of the most pertinent empirical results that measure decisive properties of real-world networks have been found in Leskovec

\footnotetext{
${ }^{1}$ We could normalize the utilities by dividing by $|V|$, and then $\epsilon$ would take values in the set $\{1 /|V|, 2 /|V|, \ldots, 1\}$. We present the theorems without the normalization so as to be consistent with all other sections.
} 
et al. $[22,24,23]$. Their focus is on tracing diffusion paths in current recommendation networks and they suggest that some of these networks have diffusion depth almost one (e.g., see Table 2 from [24]). Inspired by this, we examine the case of 2-player games, where $D(\Gamma)=k=1$.

These games may appear very simple, but still, there exist examples with no PNE, e.g., the clique of two, or more nodes ${ }^{2}$. On the other hand, given such a game, it is easy to decide whether a PNE exists in time $O\left(n^{2}\right)$. We establish the following theorem, which essentially shows that this is a well-behaved class: either PNE exist and are of a particular form, or when they do not exist we can have very good approximate PNE (in fact we can have the best possible approximation).

Theorem 8. For a game $\left(\left(G, L I S=L T M, T B C 1=R^{\prec}, T B C 2=R^{\prec}\right), \mathcal{M}, k=1\right)$, where $D=1$, either

i. all its $P N E$ have the form $\left(S_{\max }, S_{2}\right)$, where $S_{\max } \equiv \operatorname{argmax}_{S \in \mathcal{S}}\left\{\left|H_{S}\right|\right\}$, or

ii. it has no PNE but it admits a 1-PNE.

The proof as well as an analysis of the underlying structure of such networks can be found in Appendix F.

\section{Quantifying Inefficiency}

\subsection{Price of Anarchy and Stability}

Given an arbitrary $m$-player game, i.e., $\mathcal{M}=\{1, \ldots, m\}$, and a strategy profile $\mathbf{s}$, we call the sum $S W(\mathbf{s})=$ $\sum_{j=1}^{m} u_{j}(\mathbf{s})$ the social welfare of $\mathbf{s}$. The Price of Anarchy (PoA) [21], for a family of games, is defined as the worst possible ratio of $S W(\mathbf{s}) / S W\left(\mathbf{s}^{\prime}\right)$ where $\mathbf{s}$ is a social optimum, and $\mathbf{s}^{\prime}$ is a Nash equilibrium. Similarly, the Price of Stability $(\mathrm{PoS})[3]$ is defined as the best such ratio.

Suppose now that $|V|$ is sufficiently large, so that players will never play overlapping strategies at a PNE, e.g., this is ensured if $|V| \geq m k$. In that case we would have $1 \leq P o A \leq|V| /(m k)$. The question of interest then is whether PoA can be much lower than this upper bound.

The following theorem exhibits that for diffusion depths greater than one, competition can severely hurt social welfare. This can be detrimental both to the firms, and the network users, since it implies that in the worst case the firms will have a very low utility, and also the service offered by these competing products will reach only a small fraction of the nodes. This is in agreement with the worst case scenario in the model of [15] $]^{3}$ On the contrary, for the 2-player game that we examined in Section 4.4 we show that inefficiency of PNE is very low.

Theorem 9. $i$. For the family of games $\left(\left(G, L I S=L T M, T B C 1=R^{\prec}, T B C 2=R^{\prec}\right), \mathcal{M}, k\right), P o A=$ $|V| /(m k)$, and $P o S \geq \frac{k}{k+1} \frac{|V|}{m k}$, even for $D=2$.

ii. For the family of games $\left(\left(G, L I S=L T M, T B C 1=R^{\prec}, \operatorname{TBC} 2=R^{\prec}\right), \mathcal{M}=\{1,2\}, k=1\right)$, with $D=1$, we have $\operatorname{PoS}=1$, and $\operatorname{PoA} \leq S W(\mathbf{s}) /(S W(\mathbf{s})-1)$, where $\mathbf{s}$ is a social optimum. Moreover, if there exist at least two nodes with nonzero out-degree, then $\operatorname{Po} A=1$.

The proof is given in Appendix H, and the first part follows from the examples in Figure 4. The negative effect of competition on the players' utilities is further illustrated in the next subsection from the perspective of the best quality player.

\subsection{Worst-case scenarios for the best quality player}

We end our presentation with identifying a different form of inefficiency for PNE, which arises from the following question: Consider games with the reputation ordering $R^{\prec}$ as the tie-breaker. Does the firm with the best quality

\footnotetext{
${ }^{2}$ We refer the reader to Appendix F.1 for a discussion on the underlying structure of such social networks. As seen in Appendix F.1, the combination $D=k=1$ does not restrict so much the structure of the graph. E.g., under the threshold model, games in this class may be played on a star, or a clique, or any graph with a much longer diameter (but for which the thresholds and weights do not permit infections beyond the first time step).

${ }^{3}$ In the stochastic process of [15], PoA can be very high when their so-called switching function is not concave.
} 

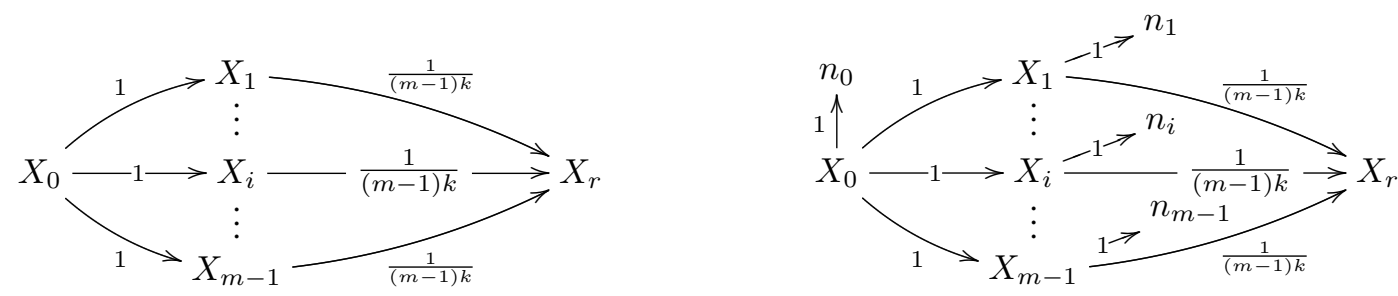

Figure 4: The social networks for the proof Theorem 9(i): As $X_{0}, \ldots, X_{m-1}$ we denote sets of $k$ nodes, whereas $X_{r}$ has an arbitrarily large number of nodes. All the underlying nodes have threshold 1.

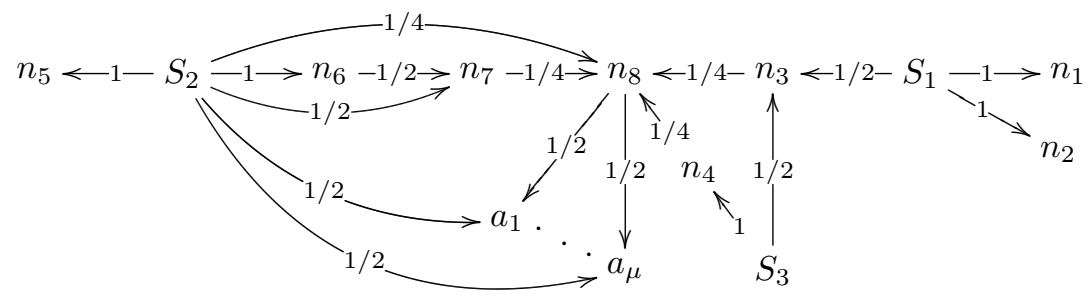

Figure 5: The network for the proof of Theorem 10(ii): All nodes have threshold 1, except of node $n_{3}$ that has $\theta_{n_{3}}=1 / 2$, and node $n_{8}$ that has $\theta_{n_{8}}=1 / 2$.

product ensure the maximum spread among all the players at any PNE? Theorem 10 illustrates that this may not always be the case for games with at least three players (but it is so for 2-player games). In fact, the payoff of the best quality player may be arbitrarily lower than the player with the highest market share at a PNE. We consider this as a form of inefficiency since in a socially desirable outcome, one would expect that the product with the best quality/reputation should have the largest market share. This surprising result dictates the necessity for quantifying such effects in PNE.

Theorem 10. Consider the class of games $\left(\left(G, L I S, T B C 1=R^{\prec}, T B C 2\right), \mathcal{M}, k\right)$.

i. If $m=2$, then for all $P N E \mathbf{s}$, it is $u_{1}(\mathbf{s}) \geq u_{2}(\mathbf{s})$.

ii. If $m \geq 3$, LIS $=L T M$, and TBC2 $=R^{\prec}$, then a game exists with a PNE $\mathbf{s}$ such that $u_{i}(\mathbf{s})<u_{j}(\mathbf{s})$, although $i \succ j$ with regard to $R^{\prec}$.

Proof. i. Assume that a PNE $\mathbf{s}=\left(S_{1}, S_{2}\right)$ exists such that $u_{1}(\mathbf{s})<u_{2}(\mathbf{s})$. Then, Player 1 can deviate to $S_{1}^{\prime}=S_{2}$, and obtain utility $u_{1}\left(S_{2}, S_{2}\right) \geq u_{2}(\mathbf{s})>u_{1}(\mathbf{s})$. Thus, $\mathbf{s}$ cannot be a PNE.

ii. Note that $R^{\prec}=1 \succ 2 \succ 3$, and consider the social network in Figure 5: As $n_{i}, \forall i \in\{1, \ldots, 8\}$, and as $a_{i}$, $\forall i \in\{1, \ldots, \mu\}$, where $\mu>k$, we denote single nodes. We assume that all of them have threshold 1 , except of nodes $n_{3}$, and $n_{8}$ that have $\theta_{n_{3}}=1 / 2$, and $\theta_{n_{8}}=1 / 2$, respectively. Moreover, as $S_{i}, \forall i \in\{1,2,3\}$, we denote sets of $k$ nodes. Finally, the edges between single nodes are annotated with their corresponding weight with regard to the LTM. On the other hand, the edges that emanate from a set $S_{i}$ are annotated with the accumulated corresponding weight of the underlying edges between each of the nodes in $S_{i}$ and the involved end-node (e.g., $\forall v \in S_{1}$, it is $w_{v n_{3}}=\theta_{n_{3}} / k$ ).

We shall prove that the strategy profile $\mathbf{s} \equiv\left(S_{1}, S_{2}, S_{3}\right)$ constitutes a PNE, even though it is $u_{2}(\mathbf{s})=$ $k+\mu+4, u_{1}(\mathbf{s})=k+3$, and $u_{3}(\mathbf{s})=k+1$-i.e., $u_{2}(\mathbf{s})>u_{1}(\mathbf{s})>u_{3}(\mathbf{s})$. Firstly, Player 1 , by deviating to $S_{3}$, receives utility $u_{1}\left(S_{3}, S_{2}, S_{3}\right)=k+3=u_{1}(\mathbf{s})$. Moreover, by deviating to $S_{2}$, he again receives utility $u_{1}\left(S_{2}, S_{2}, S_{3}\right)=k+3=u_{1}(\mathbf{s})$. Finally, Player 1 cannot achieve utility higher than $k$ by initiating any other combination of $k$ nodes from $V$. Similarly, it can be verified that neither Player 2, nor Player 3 can deviate to a better strategy, given the initial profile $\mathbf{s}$. Hence, $\mathbf{s}$ constitutes a PNE. 
In the network of Figure 5, observe that at the PNE $\mathbf{s}=\left(S_{1}, S_{2}, S_{3}\right), u_{2}(\mathbf{s})=k+\mu+4>u_{1}(\mathbf{s})+u_{3}(\mathbf{s})=2 k+4$, since $\mu>k$ - note that $\mu$ can be arbitrarily large. Thereby, if firm 1 is affiliated with firm 3 , while their products are marketed as competing and incompatible (e.g. airline merges), firm 1 is incentivized to withdraw firm 3 from the game: the resulting 2-player game between firm 1 and firm 2, has a unique PNE, namely $\left(S_{2}, S_{1}\right)$, in which firm 1 achieves the maximum possible utility $-u_{1}\left(S_{2}, S_{1}\right)=k+\mu+4$. Moreover, notice that in this 2-player game, firm 1 initiates only $k$ nodes to achieve this utility. On the other hand, in the original 3-player game, firms 1 and 3 initiate $k$ nodes each, and still they achieve a lower sum of utilities at $\mathbf{s}$.

Our discussion indicates the necessity to capture the motivation of a player in $\mathcal{M}$ to either merge with other players, or to divide itself to several new ones that, although they are affiliated, they are still non-cooperative within the induced game. For example, given the network of Figure 5 Player 1 faces the question: Should I play alone against the others, since I am the best firm, or should I merge even with the weakest? We believe this aspect of PNE is worth further investigation and we leave it as an open direction for future work.

\section{Conclusions and Future Work}

We have studied a competitive diffusion process from a non-cooperative game-theoretic viewpoint. We have investigated several aspects related to the stability of such games and we have unveiled some important parameters that to the best of our knowledge have met no previous investigation. We believe that our work motivates primarily further empirical research on social networks with regard to the following questions: Can we identify a range of typical values for decisive structural features such as the diffusion depth, the ideal spread, and the maximum diffusion collision factor? This could quantify the instability of the induced games, in light of Theorems 6 and 7, as well as the results in Section 4.2. Can we measure how much influence an individual can exert to his neighbors, so that we can have an accurate estimate of a threshold model, or any other local interaction scheme? Further research on tracing paths on recommendation networks can shed more light on this.

Other interesting questions have to do with resolving some of the remaining open problems from our work. It is still open if the complexity of determining that a PNE exists is $\Sigma_{2}^{p}$-complete, or not. The Price of Anarchy is also not yet completely determined when $D=1$ and $k$ is arbitrary. Finally, additional compelling questions may concern the robustness to network changes. For example, how does the introduction of new individuals in the network affect any of the properties we studied?

\section{References}

[1] N. Alon, M. Feldman, A. D. Procaccia, and M. Tennenholtz. A note on competitive diffusion through social networks. Information Processing Letters (IPL), 110(6):221-225, 2010.

[2] N. Alon, M. Feldman, A. D. Procaccia, and M. Tennenholtz. Erratum: A note on competitive diffusion through social networks. http://www.cs.cmu.edu/ arielpro/papers/diffusion.err. pdf, 2011.

[3] E. Anshelevich, A. Dasgupta, J. Kleinberg, E. Tardos, T. Wexler, and T. Roughgarden. The price of stability for network design with fair cost allocation. In Proceedings of 45th Symposium on Foundations of Computer Science (FOCS), pages 295-304, 2004.

[4] K. R. Apt and E. Markakis. Diffusion in social networks with competing products. In 4th International Symposium on Algorithmic Game Theory (SAGT), pages 212-223, 2011.

[5] A.-L. Barabási and R. Albert. Emergence of scaling in random networks. Science, 286:509-512, 1999.

[6] S. Bharathi, D. Kempe, and M. Salek. Competitive influence maximization in social networks. In International Workshop on Internet and Network Economics (WINE), pages 306-311, 2007.

[7] A. Borodin, Y. Filmus, and J. Oren. Threshold models for competitive influence in social networks. In 6th International Workshop on Internet and Network Economics (WINE), pages 539-550, 2010. 
[8] T. Carnes, C. Nagarajan, S. M. Wild, and A. van Zuylen. Maximizing influence in a competitive social network: A follower's perspective. In 9th International Conference on Electronic Commerce (ICEC), pages 351-360, 2007.

[9] N. Chen. On the approximability of influence in social networks. SIAM Journal on Discrete Mathematics (SIDMA), 23(3):1400-1415, 2009.

[10] S. Chien and A. Sinclair. Convergence to approximate Nash equilibria in congestion games. Games and Economic Behavior, 71(2):315-327, 2011.

[11] P. Domingos and M. Richardson. Mining the network value of customers. In 7th ACM SIGKDD International Conference on Knowledge Discovery and Data Mining (KDD), pages 57-66, 2001.

[12] D. Easley and J. Kleinberg. Networks, Crowds, and Markets. Cambridge University Press, 2010.

[13] J. Goldenberg, B. Libai, and E. Muller. Talk of the network: A complex systems look at the underlying process of word-of-mouth. Marketing Letters, 12(3):211-223, 2001.

[14] S. Goyal. Connections: An introduction to the economics of networks. Princeton University Press, 2007.

[15] S. Goyal and M. Kearns. Competitive contagion in networks. In 44th ACM Symposium on Theory of Computing (STOC), pages 759-774, 2012.

[16] M. S. Granovetter. Threshold models of collective behavior. The American Journal of Sociology, 83(6):1420-1443, 1978.

[17] N. Immorlica, J. Kleinberg, M. Mahdian, and T. Wexler. The role of compatibility in the diffusion of technologies through social networks. In Proceedings of the 8th ACM Conference on Electronic Commerce (EC), pages 75-83, 2007.

[18] M. Jackson. Social and Economic Networks. Princeton University Press, 2008.

[19] D. Kempe, J. Kleinberg, and E. Tardos. Maximizing the spread of influence in a social network. In 9th ACM SIGKDD International Conference on Knowledge Discovery and Data Mining (KDD), pages 137146, 2003.

[20] J. Kostka, O. Y. A., and R. Wattenhofer. Word of mouth: Rumor dissemination in social networks. In 15th International Colloquium on Structural Information and Communication Complexity (SIROCCO), pages 185-196, 2008.

[21] E. Koutsoupias and C. H. Papadimitriou. Worst case equilibria. In Annual IEEE Symposium on Theoretical Aspects of Computer Science (STACS), pages 404-413, 1999.

[22] J. Leskovec, L. Adamic, and B. Huberman. The dynamics of viral marketing. ACM Transactions on the Web (TWEB), 1(1):Article No. 5, 2007.

[23] J. Leskovec, M. McGlohon, C. Faloutsos, N. Glance, and M. Hurst. Cascading behavior in large blog graphs. In SIAM International Conference on Data Mining (SDM), pages 551-556, 2007.

[24] J. Leskovec, A. Singh, and J. Kleinberg. Patterns of influence in a recommendation network. In Pacific-Asia Conference on Knowledge Discovery and Data Mining (PAKDD), pages 380-389, 2006.

[25] D. Monderer and L. S. Shapley. Potential games. Games and Economic Behavior, 14:124-143, 1996.

[26] S. Morris. Contagion. The Review of Economic Studies, 67(1):57-78, 2000.

[27] E. Mossel and S. Roch. On the submodularity of influence in social networks. In Symposium on Theory of Computing (STOC), pages 128-134, 2007.

[28] M. E. J. Newman, S. H. Strogatz, and D. J. Watts. Random graphs with arbitrary degree distributions and their applications. Physical Review E, 64(2):026118, August 2001.

[29] T. C. Schelling. Micromotives and Macrobehavior. Norton, New York, NY, 1978. 
[30] S. Simon and K. R. Apt. Choosing products in social networks. Manuscript, available at http: / / arxiv . org/abs/1202.2209, 2012.

[31] R. Takehara, M. Hachimori, and M. Shigeno. A comment on pure-strategy Nash equilibria in competitive diffusion games. Information Processing Letters (IPL), 112(3):59-60, 2010.

[32] M. Voorneveld and H. Norde. A characterization of ordinal potential games. Games and Economic Behavior, 19:235-242, 1997. 


\section{A Illustrative examples.}

Example 4 (Illustrative example over the definitions in the Preliminaries). Let the game $(\mathscr{N}, \mathcal{M}, k=1)$ over the social network $\left(G, L I S=L T M, T B C 1=R^{\prec}, T B C 2=R^{\prec}\right)$ in Figure 6: As $n_{i}, \forall i \in\{1, \ldots, 5\}$, we denote single nodes. Further, we assume that all of them have threshold one, except $n_{3}$ that has $1 / 2$; i.e., $\theta_{n_{i}}=1, \forall i \in\{1,2,4,5\}$, and $\theta_{n_{3}}=1 / 2$. Moreover, the edges $\left(n_{i}, n_{j}\right)$, where $i, j \in\{1, \ldots, 5\}$, are annotated with their corresponding weight.

Now, verify that the in-neighbors of, e.g., $n_{4}$ form the set $N\left(n_{4}\right)=\left\{n_{2}, n_{3}\right\}$, while for $n_{1}$ it is $N\left(n_{1}\right)=\emptyset$. Further, observe that the diffusion depth is $D=2$. Additionally, the ideal spread of $n_{2}$ is $H_{n_{2}}=\left\{n_{2}, n_{3}, n_{4}\right\}$, i.e., $n_{3}$, and $n_{4}$ are both reachable by $n_{2}$. On the other hand, $n_{4}$ is not reachable from $n_{1}$, since $w_{n_{3} n_{4}}=1 / 2<$ $\theta_{n_{4}}=1$, and it is $H_{n_{1}}=\left\{n_{3}, n_{5}\right\}$. Also, the ideal spread of $n_{5}$ is $H_{n_{5}}=\left\{n_{5}\right\}$.

Next, we illustrate Definition 4, and specifically the terms $\alpha_{i}(\mathbf{s}), \beta_{i}(\mathbf{s}), \gamma_{i}(\mathbf{s})$, where $i \in\{1,2\}$, and $\mathbf{s} \in \mathcal{S}^{2}$ : Observe that $n_{4}$ can be infected during the diffusion process by a player $i \in \mathcal{M}$, only if $i$ has colored both nodes $n_{2}$ and $n_{3}$ - either at the initiation step, or later. Therefore, if the strategy profile $\mathbf{s}=\left(n_{1}, n_{2}\right)$ is played, node $n_{4}$ remains white after the termination of the competitive diffusion process: Player 1 infects first node $n_{3}$, according to TBC2 $=R^{\prec}$ - thus, it also is $\beta_{2}(\mathbf{s})=1$. As a result, given that Player 1 initiates $n_{1}$ and Player 2 initiates $n_{2}$, it is $\gamma_{2}(\mathbf{s})=1$, since firm 2 never becomes eligible to infect $n_{4}$. On the other hand, it is $\gamma_{1}(\mathbf{s})=0$. Clearly, $\gamma_{1}(\mathbf{s}) \neq \gamma_{2}(\mathbf{s})$. Similarly, $\alpha_{1}(\mathbf{s})=0, \alpha_{2}(\mathbf{s})=0, \beta_{1}(\mathbf{s})=0$. Thereby, $u_{1}(\mathbf{s})=\left|H_{n_{1}}\right|-\alpha_{1}(\mathbf{s})-\gamma_{1}(\mathbf{s})=3-0-0=3$, while $u_{2}(\mathbf{s})=\left|H_{n_{2}}\right|-\alpha_{2}(\mathbf{s})-\beta_{2}(\mathbf{s})-\gamma_{2}(\mathbf{s})=3-0-1-1=1$.

Finally, if both players choose to initiate the same node, e.g., $n_{1}$, then node $n_{1}$ will be colored only by Player 1 , according to TBC1 $=R^{\prec}$, and it will be $u_{1}\left(n_{1}, n_{1}\right)=\left|H_{n_{1}}\right|-\alpha_{1}\left(n_{1}, n_{1}\right)-\gamma_{1}\left(n_{1}, n_{1}\right)=3-0-0=3$, while $u_{2}\left(n_{1}, n_{1}\right)=\left|H_{n_{1}}\right|-\alpha_{2}\left(n_{1}, n_{1}\right)-\beta_{2}\left(n_{1}, n_{1}\right)-\gamma_{2}\left(n_{1}, n_{1}\right)=3-0-1-2=0$ (note that $\gamma_{2}\left(n_{1}, n_{1}\right)=2$ because firm 2 never becomes eligible to infect $n_{3}$ and $n_{5}$, since it did not manage to color $\left.n_{1}\right)$.

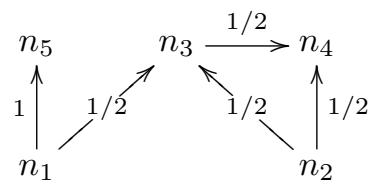

Figure 6: A social network $\left(G, \mathrm{LIS}=\mathrm{LTM}, \operatorname{TBC} 1=R^{\prec}, \mathrm{TBC} 2=R^{\prec}\right)$.

Example 5 (Introductory example for Theorem 5). Consider a game $\left(\left(G, L I S=L T M, T B C 1=R^{\prec}, T B C 2\right)\right.$, $\mathcal{M}, k=1)$ over the social network of Figure 7. We assume that all the nodes have threshold 1.

Next, verify that $\left|H_{S_{n_{1}}}\right|=5$, whereas $\left|H_{S_{n_{2}}}\right|=3$. Now, observe that $\left|H_{S_{n_{2}}}\right|>\left|H_{S_{n_{1}}}\right| / 2$, and as a result $\left|H_{S_{n_{2}}}\right|=3>\left|H_{S_{n_{1}}}\right|-\left|H_{S_{n_{2}}}\right|=2$. Therefore, if Player 1 initiates node $n_{1}$, and Player 2 node $n_{2}$, then the latter player will eventually achieve a higher utility than the former. On the other hand, if Player 1 initiates $n_{2}$, then Player 2 would prefer to initiate $n_{1}$. And then, Player 1 would prefer again to play $n_{1}$. As a result, the following improvement cycle exists (we annotate each arrow with the number of the player that diverges to a better strategy): $\left(S_{1}=n_{1}, S_{2}=n_{1}\right) \stackrel{2}{\rightarrow}\left(n_{1}, n_{2}\right) \stackrel{1}{\rightarrow}\left(n_{2}, n_{2}\right) \stackrel{2}{\rightarrow}\left(n_{2}, n_{1}\right) \stackrel{1}{\rightarrow}\left(n_{1}, n_{1}\right)$.

In other words, this game does not admit a generalized ordinal potential since the strategy with the second highest ideal spread can first infect more than half the number of nodes that the strategy with the highest ideal spread also can.

On the other hand, if we consider the full and complete version of the above tree, i.e., by adding two children, $n_{6}$ and $n_{7}$, to node $n_{3}$, it can be seen that the new game admits a generalized ordinal potential, even though the condition of Theorem 5 does not hold (since then $\left|H_{n_{2}}\right|=\left|H_{n_{3}}\right|$ ). Moreover, this example can be generalized to Corollary 4, using similar arguments as in the proof of Theorem 5. Therefore, in some cases, the conditions of Theorem 5 can be relaxed. 


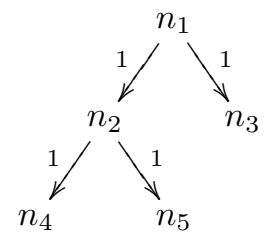

Figure 7: Illustrative example for Theorem 5.

\section{B Section 3: Proof of Theorem 1.}

co-NP-hardness. We provide a reduction from 3SAT. To this goal, given a 3CNF formula $\Phi$ with $n$ variables and $m$ clauses, where we assume that $n>6$, we construct a social network $\left(G\right.$, LIS $=$ LTM, TBC1 $=R^{\prec}$, $\mathrm{TBC} 2=R^{\prec}$ ) that consists of two basic parts. The first part (Figure 8a) consists of $n$ components with dummy nodes and has $n$ dummy nodes of type $a$ and $n$ dummy nodes of type $b$. Node $a_{i}$ connects to node $b_{i}$ for $i=1,2, \ldots, n$. The second part (Figures $8 \mathrm{~b}, 8 \mathrm{c}$ ) is determined by the formula $\Phi$. It consists of literal nodes, variable nodes, clause nodes, one extra node, that we call node $c$ and dummy nodes of type $d$. Every formula's literal $x_{i}$, or $\neg x_{i}$ is connected to variable node $v_{i}$. If a formula's clause contains a literal then there is an edge from the literal to the clause. All clause, variable and literal nodes connect to node $c$ with an edge of weight $\frac{1}{3 n+m}$. Node $c$ connects to $M=2+\left\lfloor\frac{m}{2}\right\rfloor$ dummy nodes of type $d$. The threshold of any clause node is $1 / 3$, which is also equal to the weight of an edge from a literal node to a clause. Also the threshold of any variable node is $1 / 2$, and equal to the weight of every edge from a literal to a variable. Every unspecified weight has value 1 and every node has threshold 1 except for $c$ that has $\frac{2 n+m}{3 n+m}$. It is easy to see that this construction needs polynomial time. Finally we define a game with $k=n$.

We first establish some properties for the strategy space of the game.

Lemma 4. If a player does not choose $c$ as part of his strategy then the only possible way to win $c$ is by choosing the corresponding literal nodes of a satisfying truth assignment of the formula (if there exists one).

Proof. A node will be called a formula node if it is either a literal, or variable, or clause node. From the game's structure, the player has to win at least $2 n+m$ formula nodes. Suppose he chooses $n_{1}$ literal nodes and $n_{2}$ variable nodes. Then he will win at most $2 n_{1}+n_{2}+m$ formula nodes. Since $n_{1}+n_{2}$ is at most $n$ the expression is maximized for $n_{1}=n$ giving an upper bound of $2 n+m$ formula nodes. Hence the player should choose only literal nodes. Since $k=n$, if the player chooses the two opposite literals of a variable then there will be one variable that cannot be won and thus, he will win less than $2 n+m$ formula nodes. Hence he needs to choose exactly one literal from each variable. If these literal nodes do not connect to all clause nodes then he will also get less than $2 n+m$ formula nodes. So his strategy corresponds to a satisfying truth assignment.

We now argue that there is no point in playing strategy profiles that contain nodes with out-degree equal to 0 as well as variable and clause nodes. Let $A$ be the following set of nodes $\left\{x_{1}, \neg x_{1}, x_{2}, \neg x_{2}, \ldots, x_{n}, \neg x_{n}\right.$, $\left.a_{1}, a_{2}, \ldots, a_{n}, c\right\}$. We say that a strategy $S$ is reasonable, or that a player plays reasonably if $S \subseteq A$.

Lemma 5. Any unreasonable strategy is strictly dominated for both players.

Proof. We argue first for player 1. Consider an arbitrary strategy profile $\mathbf{s}=\left(S_{1}, S_{2}\right)$ such that $S_{1}$ contains a node $y$ with $y \notin A$. We show that it is strictly better for the player to replace $y$ with some node from $A$. There are four cases to consider for $y$.

Case 1: $y$ is of type $b$. Let $y=b_{i}$ for some $i$. In that case, the player wins only the node itself by playing $y$. If the corresponding $a_{i}$ node is not played under $s$, and node $b_{i}$ is not included in $S_{2}$ either, then the player can switch to $a_{i}$ and he will win an extra node this way. Hence suppose that this is not the case. If there is any pair $\left(a_{j}, b_{j}\right)$ such that they are both free, then he has an incentive to play $a_{j}$ and then he will also win $b_{j}$. Assume therefore that there is no free pair $\left(a_{j}, b_{j}\right)$. This means that there is at least one used node in every dummy component (and there were 2 nodes used in the component $\left(a_{i}, b_{i}\right)$. Thus there are at most $n-1$ nodes used in the rest of 


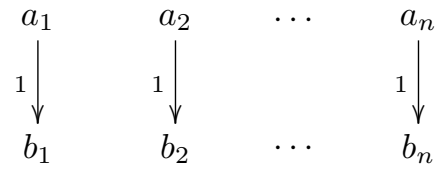

(a) The first part of the constructed social network.

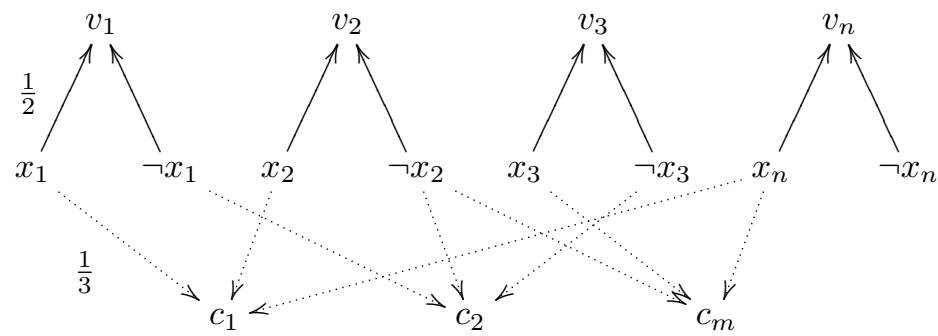

(b) The upper component of the second part of the constructed social network. We depict an instance, given a boolean formula $\Phi=$ $\left(x_{1} \vee x_{2} \vee x_{n}\right) \wedge\left(\neg x_{1} \vee \neg x_{2} \vee \neg x_{3}\right) \wedge\left(\neg x_{2} \vee x_{3} \vee x_{n}\right)$. All the edges from a literal to a clause node have weight $1 / 3$, and all the edges from a literal to a variable node have weight $1 / 2$. Finally, observe that the edges from all these clause, variable and literal nodes to node $c$ of Figure $8 \mathrm{c}$ are not depicted.

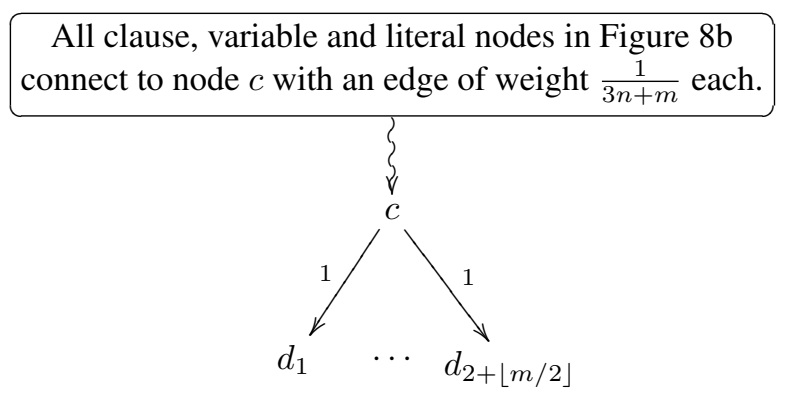

(c) Overview of the second part of the constructed social network. The curly edge denotes that all clause, variable and literal nodes in Figure $8 \mathrm{~b}$ connect to node $c$ with an edge of weight $\frac{1}{3 n+m}$ each.

Figure 8: The constructed social network $\left(\left(G, \mathrm{LIS}=\mathrm{LTM}, \mathrm{TBC} 1=R^{\prec}, \mathrm{TBC} 2=R^{\prec}\right)\right.$ for the proof of Theorem 1. 
the graph. This means that at least one triplet $\left(v_{j}, x_{j}, \neg x_{j}\right)$ is free and the player can play e.g. $x_{i}$ to win an extra node.

Case 2: $y$ is of type $d$, say $y=d_{i}$. Then again, if there is a free pair $\left(a_{j}, b_{j}\right)$ he can switch to $a_{j}$. Otherwise there are at least $n$ occupied nodes in the dummy components. Then either one triplet $\left(v_{j}, x_{j}, \neg x_{j}\right)$ is free, or node $c$ is free, hence the player has a strictly better strategy.

Case 3: $y$ is a variable node, $y=v_{i}$. A variable node has a nonzero out-degree, however it only connects to $c$ and as we saw in Lemma 4 the only way to infect $c$ is by playing only the literals. Thus we only gain node $v_{i}$ by using it as part of the strategy $S_{1}$. If the nodes $\left(v_{i}, x_{i}, \neg x_{i}\right)$ are not used by the other player, or the first player does not use $x_{i}, \neg x_{i}$ in $S_{1}$, then he can switch to $x_{i}$ and win more. Suppose this is not the case and suppose also that all other triplets $\left(v_{j}, x_{j}, \neg x_{j}\right)$ are used. Then there is at least one free pair $\left(a_{j}, b_{j}\right)$ and he can switch to that.

Case 4: $y$ is a clause node, $y=c_{i}$. Then a similar reasoning applies as with Case 3. A clause node as part of a strategy only grants the player the node itself. If there is a free triplet $\left(v_{j}, x_{j}, \neg x_{j}\right)$ then he can switch to $x_{i}$ and win more. Otherwise, either there is at least one free pair $\left(a_{j}, b_{j}\right)$ and he can switch to that, or node $c$ is free.

For the second player, the argument is almost identical and we omit it.

Since a strictly dominated strategy cannot be used in an equilibrium, we have established that if there exists a Nash equilibrium $\mathbf{s}=\left(S_{1}, S_{2}\right)$, then both $S_{1}$ and $S_{2}$ are reasonable. Furthermore, the following is also easy to see and based on similar arguments:

Lemma 6. Any strategy $S$, in which $\left\{x_{i}, \neg x_{i}\right\} \subseteq S$, for some $i \in\{1, \ldots, n\}$ is strictly dominated for either player.

We now proceed to show that our game does not have a PNE if and only if $\Phi$ is satisfiable.

Suppose $\Phi$ is not satisfiable. We describe the following strategies for the two players: the first player plays as if he is alone, choosing the best $n$ nodes for him. With a slight modification of Lemma 5 we can show that he plays reasonably. Afterwards the second player plays optimally, taking into consideration the first player's strategy. Again from Lemma 5 we know that he plays reasonably. Let $\mathbf{s}=\left(S_{1}, S_{2}\right)$ be the resulting profile. We argue that this pair of strategies forms a PNE. The second player by construction does not have an incentive to change his strategy. The first player would have an incentive to change only if he loses some nodes from the second player that belong to the cumulative ideal spread $H_{S_{1}}$ when he plays alone. Since both play reasonably, and the first player has priority, he cannot lose any nodes of type $a$, or a literal node, and furthermore he cannot lose any variable, or clause nodes either. The only node that he could lose is $c$ and this happens only if the first player does not play $c$ and if $c \in H_{S_{1}}$. But then by Lemma 4, the first player has targeted a satisfying assignment of $\Phi$, a contradiction.

Suppose that $\Phi$ is satisfiable. Let us assume that there exists a PNE $\mathbf{s}=\left(S_{1}, S_{2}\right)$. From Lemma 5 both players play reasonably. We consider the following cases:

- Node $c$ is played by the first player. Then the second player will have to play in $A-\{c\}$ and be at least two diffusion steps away from $c$. To calculate the utility of the first player, we see that he plays at most $n-1$ literal nodes, which grant him an infection of at most $n-1$ variable nodes as well as at most the clauses (or he plays nodes of type $a$ which grant him only one extra node). Thus the total utility of the first player is upper bounded by:

$$
1+2+\left\lceil\frac{m}{2}\right\rceil+2(n-1)+m=1+m+\left\lceil\frac{m}{2}\right\rceil+2 n
$$

Hence the first player will have an incentive to leave $c$ and pick the literal nodes that satisfy the formula (since he will also be two steps away from $c$ and has priority over the second player). Then his utility would be:

$$
3+\left\lceil\frac{m}{2}\right\rceil+2 n+m
$$

which is bigger than before, a contradiction to the fact that the strategy profile is a PNE.

- Node $c$ is not played by the first player. Then there are three possible choices for the first player. The first is to play only nodes of type $a$, the second is to play only literal nodes and by Lemma 6 , he would play the 
satisfying literals of $\Phi$, and the third is to play a mixture of some literal nodes and some nodes of type $a$. The first choice however is not possible because he would win only $2 n$ nodes and he could easily switch to infecting the satisfying literals of $\Phi$. Hence $s$ would not be a PNE. For the second and third choices, we claim that the second player has to play $c$. Consider for example the second choice. In that case, if the second player does not play $c$, then by playing reasonably he can win at most an extra node for each node that he chooses. So he has an incentive to exchange a node for $c$ and win the extra $M$ nodes of type $d$. For the third case, if the second player does not play $c$, then he can win at most $2 n+r$ nodes, where $r$ is the number of clauses connected with the literals he chose in his strategy. We claim that there exists one literal node that connects to at most $\left\lfloor\frac{m}{2}\right\rfloor$ clauses. This follows because there exists one variable that occurs in at most $\left\lfloor\frac{m}{2}\right\rfloor$ clauses for any 3CNF formula with $n>6$. Now if neither player played $c$, one literal node is played for every variable of the formula. But if the specific literal under consideration is played by any of the players, then s would not be a PNE, contrary to what we assumed because they could replace this literal node with $c$ and win the extra $2+\left\lfloor\frac{m}{2}\right\rfloor$ nodes. Since we assumed that the first player does not play $c$, the only possible choice left is that the second player plays $c$.

Hence, we have concluded that the second player has to choose $c$. But then the maximum utility of the first player is $2 n+m$. Since the formula is satisfiable there are $n-1$ literals of different variables that satisfy at least $\left\lceil\frac{m}{2}\right\rceil$ clauses. Otherwise, if this were not true, every literal would connect to at least $\left\lceil\frac{m}{2}\right\rceil$ clause nodes and that means that the total in degree of the clause nodes is at least $n\left\lceil\frac{m}{2}\right\rceil$. But this cannot happen since $n>6$ and each one of the $m$ clauses has exactly three literals. By choosing these $n-1$ literal nodes and $c$ the first player will win $2(n-1)+\left\lfloor\frac{m}{2}\right\rfloor+1+2+\left\lceil\frac{m}{2}\right\rceil=2 n+1+m>2 n+m$ nodes.

Therefore the formula is satisfiable if and only if there exists no PNE, and this concludes the proof.

Proof of membership in $\Sigma_{2}^{p}$. We need to show that our problem belongs to $N P^{N P}$. Our problem can be stated in the form:

$$
\exists \text { strategy profile } \mathbf{s}=\left(S_{1}, S_{2}\right): \forall \text { unilateral deviation is not profitable }
$$

For the first quantifier, we can simply guess a strategy profile s. As for the second quantifier, it suffices to observe that it is the negation of an NP statement. To show that no deviation is profitable, we can use an oracle for NP as follows: we can solve the negation which says that there exists a deviation that is profitable for some player. This can be solved in NP by guessing a deviation and checking that the player who deviates is doing strictly better. This means that we can determine with an NP oracle whether all deviations are not profitable.

\section{Section 4.1: Proofs of Theorem 2, and Corollary 1.}

Proof of Theorem 2. We construct a certain class of graphs, denoted by $\mathcal{G}_{\mathcal{D}}$, that induce a class of social networks with the involved property. At this point, we stress that for the games in question it always is $u_{2}(S, S)=0$, $\forall S \in \mathcal{S}$, while $u_{2}\left(S_{1}, S_{2}\right) \geq 1, \forall S_{1}, S_{2} \in \mathcal{S}$ such that $S_{1} \neq S_{2}$ - i.e., given a strategy profile $(S, S) \in \mathcal{S}$ Player 2 always wants to deviate to any strategy $S_{2} \neq S$. Therefore, we prove that any set of consistent improvement paths and cycles is realizable, given that it obey to this previous restriction.

To this end, the following definitions are necessary.

Definition 7. $i$. Assume that only one player from $\mathcal{M}$ participates in the game, and let $S \in \mathcal{S}$ be one of its strategies. We define as ideal spread of $\mathbf{S}$ at the time step $\mathrm{t}$ of the diffusion process, denoted by $I_{S}^{t}$, the set of nodes in $V$ that adopt the associated with this player color under strategy $S$ and at the time step tonly. Further, we set $I_{S}^{0} \equiv S$.

ii. Assume that only one player from $\mathcal{M}$ participates in the game, and let $S \in \mathcal{S}$ be one of its strategies. We define as cumulative ideal spread of $\mathbf{S}$ at time step $\mathbf{t}$ of the diffusion process, denoted by $H_{S}^{t}$, the set of nodes in $V$ that adopt the associated with this player color under strategy $S$ and until the time step $t$, i.e., $H_{S}^{t} \equiv \bigcup_{i=0}^{t} I_{S}^{i}$.

Next, we set $\mathcal{S}_{\mathcal{D}}=\left\{S_{1}, \ldots, S_{r}\right\}$. Moreover, we note that given a strategy profile $\mathbf{s}=\left(S_{x}, S_{y}\right) \in \mathcal{S}^{2},(x, y) \in$ $\{1, \ldots,|\mathcal{S}|\}^{2}$, we denote the players' utilities as $u_{i}^{x y}$, instead of $u_{i}\left(S_{x}, S_{y}\right), \forall i \in \mathcal{M}=\{1,2\}$. 


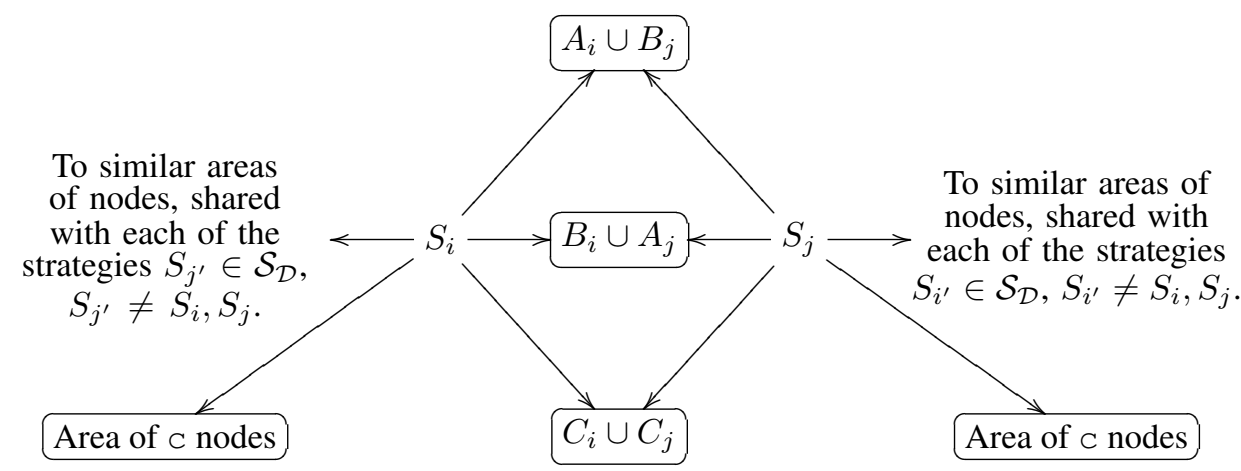

Figure 9: The partially shared areas $A_{i} \cup B_{j}, B_{i} \cup A_{j}$, and $C_{i} \cup C_{j}$ between the two strategies $\left(S_{i}, S_{j}\right) \in \mathcal{S}_{\mathcal{D}}{ }^{2}$, $S_{i} \neq S_{j}$. Moreover, the similar areas that exists between $S_{i}, S_{j}$ and any other strategy in $\mathcal{S}_{\mathcal{D}}{ }^{2}$ are depicted, as well as, the sets of $\mathrm{c}$ nodes that each of them targets.

Now, we continue with the main part of the proof, where we consider without loss of generality that $k=$ 1 , and $D=2$. Moreover, we assume that under our construction it holds $\alpha_{1}\left(S_{1}, S_{2}\right)=\alpha_{2}\left(S_{2}, S_{1}\right)$, and $\beta_{2}\left(S_{1}, S_{2}\right)=\beta_{2}\left(S_{2}, S_{1}\right), \forall\left(S_{1}, S_{2}\right) \in \mathcal{S}$ (Definition 4). Thereby, we set for simplicity $\alpha_{S_{2} S_{1}} \equiv \alpha_{1}\left(S_{1}, S_{2}\right)$, i.e., $\alpha_{S_{1} S_{2}}=\alpha_{2}\left(S_{1}, S_{2}\right)$.

Additionally, we assume that $\operatorname{LIS}=\operatorname{LTM}\left(w_{u v} \geq \theta_{v}, \forall(u, v) \in E\right)$. Therefore, for $i \in\{1,2\}$, it is $\gamma_{i}\left(S_{1}, S_{2}\right)=$ $0, \forall\left(S_{1}, S_{2}\right) \in \mathcal{S}^{2}$, where $S_{1} \neq S_{2}$. Also, recall that $\gamma_{1}(S, S)=0, \forall S \in \mathcal{S}$, since TBC1 $=R^{\prec}$.

Furthermore, we let $\left|H_{S_{i}}\right|=H, \forall i \in\{1, \ldots, r\}$, where $H$ will be appropriately chosen to guarantee that the set $\mathcal{S}_{\mathcal{D}}$ is a sink in $\mathcal{S}$.

Next, we assume for the constructed class of social networks that:

i. $I_{S_{i}}^{0} \cap I_{S_{j}}^{1}=\emptyset, \forall(i, j) \in\{1, \ldots, r\}^{2}$, where $i \neq j$,

ii. $\left(H_{S_{j}}^{1} \cap I_{S_{i}}^{2}\right) \cap\left(H_{S_{j^{\prime}}}^{1} \cap I_{S_{i^{\prime}}}^{2}\right)=\emptyset, \forall\left(i, j, i^{\prime}, j^{\prime}\right) \in\{1, \ldots, r\}^{4}$, where $(i, j) \neq\left(i^{\prime}, j^{\prime}\right)$,

iii. $\left(H_{S_{j}}^{1} \cap H_{S_{i}}^{1}\right) \cap\left(H_{S_{j^{\prime}}}^{1} \cap H_{S_{i^{\prime}}}^{1}\right)=\emptyset, \forall\left(i, j, i^{\prime}, j^{\prime}\right) \in\{1, \ldots, r\}^{4}$, where $(i, j) \neq\left(i^{\prime}, j^{\prime}\right)$,

iv. $\left(H_{S_{j}}^{1} \cap H_{S_{i}}^{1}\right) \cap\left(I_{S_{j^{\prime}}}^{2} \cap I_{S_{i^{\prime}}}^{2}\right)=\emptyset, \forall\left(i, j, i^{\prime}, j^{\prime}\right) \in\{1, \ldots, r\}^{4}$, where $(i, j) \neq\left(i^{\prime}, j^{\prime}\right)$,

v. $\left(I_{S_{j}}^{2} \cap I_{S_{i}}^{2}\right) \cap\left(I_{S_{j^{\prime}}}^{2} \cap I_{S_{i^{\prime}}}^{2}\right)=\emptyset, \forall\left(i, j, i^{\prime}, j^{\prime}\right) \in\{1, \ldots, r\}^{4}$, where $(i, j) \neq\left(i^{\prime}, j^{\prime}\right)$, as well as,

vi. $H=(r-1)\left(2 \alpha_{\max }+\beta_{\max }\right)+1+\mathrm{c}$, where c is appropriately chosen for the set $\mathcal{S}_{\mathcal{D}}$ to be a sink in $\mathcal{S}$.

Over these assumptions, we can construct a class of social networks, where $H_{S_{j}}^{1} \cap I_{S_{i}}^{2}, H_{S_{j}}^{1} \cap H_{S_{i}}^{1}$, and $I_{S_{j}}^{2} \cap I_{S_{i}}^{2}$ can be independently decided from one another. Particularly, without loss of generality, consider the pair of strategies $\left(S_{i}, S_{j}\right)$, where $i \neq j$, and $(i, j) \in\{1, \ldots, r\}$. Then, recall that LIS $=\operatorname{LTM}\left(w_{u v} \geq \theta_{v}, \forall(u, v) \in E\right)$, and consider the following three disjoint areas of $H_{S_{i}}$ that can share nodes only with three corresponding areas of $H_{S_{j}}$ (Figure 9):

A. Name this area $A_{i}$ (Figure 10); through its structure we shall later show that the value of $\alpha_{S_{i} S_{j}}$ can be independently decided from the values of $\alpha_{S_{i^{\prime}} S_{j^{\prime}}}, \beta_{2}\left(S_{i^{\prime}}, S_{j^{\prime}}\right), \forall\left(i^{\prime}, j^{\prime}\right) \in\{1, \ldots, r\}^{2}$. Assume, $A_{i} \subset H_{S_{i}}$, $\left|A_{i}\right|=\alpha_{\max }$, and that $\forall k \in A_{i},\left(S_{i}, k\right) \in E$, while $\nexists v \in V \backslash\left\{S_{i}, S_{j}\right\}:(v, k) \in E$. Additionally, assume $A_{i}=\left(H_{S_{i}}^{1} \cap I_{S_{j}}^{2}\right) \cup X_{i}$, where $X_{i} \equiv A_{i} \backslash\left(H_{S_{i}}^{1} \cap I_{S_{j}}^{2}\right)$, and that $\forall k \in X_{i}, \nexists v \in V:(k, v) \in E$. Similarly, $\forall k \in H_{S_{i}}^{1} \cap I_{S_{j}}^{2}, \nexists v \in V:(k, v) \in E$. Next, note that it is $\left|H_{S_{i}}^{1} \cap I_{S_{j}}^{2}\right|=\alpha_{S_{i} S_{j}}$ by definition, therefore $\left|X_{i}\right|=\alpha_{\max }-\alpha_{S_{i} S_{j}}$.

The corresponding region in $H_{S_{j}}$ subset of which is the set $H_{S_{i}}^{1} \cap I_{S_{j}}^{2}$ is the area $B_{j}$ (Figure 10). Below we define $B_{i}$. 


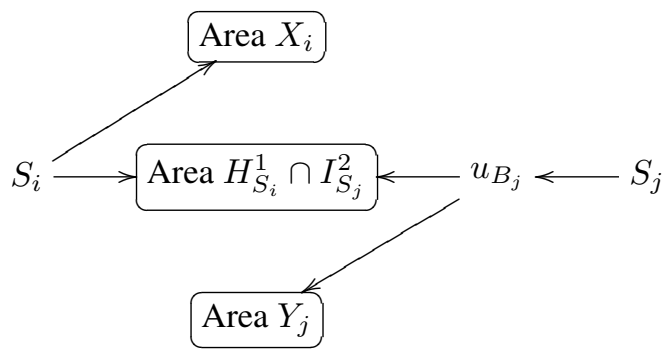

Figure 10: The partially shared area $A_{i} \cup B_{j}$ between the two strategies $\left(S_{i}, S_{j}\right) \in \mathcal{S}_{\mathcal{D}}{ }^{2}, S_{i} \neq S_{j}: A_{i} \equiv$ $\left(H_{S_{i}}^{1} \cap I_{S_{j}}^{2}\right) \cup X_{i}$, and $B_{j} \equiv\left(H_{S_{i}}^{1} \cap I_{S_{j}}^{2}\right) \cup Y_{j} \cup\left\{u_{B_{j}}\right\}$. Moreover, note that $\left|H_{S_{i}}^{1} \cap I_{S_{j}}^{2}\right|=\alpha_{S_{i} S_{j}}$, and $\left|X_{i}\right|=\alpha_{\max }-\alpha_{S_{i} S_{j}}$. Therefore, $\left|A_{i}\right|=\alpha_{\max }$. Also, it is $\left|Y_{j}\right|=\alpha_{\max }-\alpha_{S_{i} S_{j}}$, and as a result $\left|B_{j}\right|=\alpha_{\max }+1$.

B. Name this part $B_{i}$ (Figure 11); through its structure we shall later show that the value of $\alpha_{S_{j} S_{i}}$ can be independently decided from the values of $\alpha_{S_{i^{\prime}} S_{j^{\prime}}}, \beta_{2}\left(S_{i^{\prime}}, S_{j^{\prime}}\right), \forall\left(i^{\prime}, j^{\prime}\right) \in\{1, \ldots, r\}^{2}$. Assume, $B_{i} \subset H_{S_{i}}$, $\left|B_{i}\right|=1+\alpha_{\max }$. Specifically, let $B_{i}=\left(H_{S_{j}}^{1} \cap I_{S_{i}}^{2}\right) \cup Y_{i} \cup\left\{u_{B_{i}}\right\}$, where $Y_{i} \equiv B_{i} \backslash\left(H_{S_{j}}^{1} \cap I_{S_{i}}^{2}\right) \cup\left\{u_{B_{i}}\right\}$, and $u_{B_{i}}$ denotes a single node such that $\left(S_{i}, u_{B_{i}}\right) \in E$. Moreover, $\forall k \in\left(H_{S_{j}}^{1} \cap I_{S_{i}}^{2}\right) \cup Y_{i}$ we consider $\left(u_{B_{i}}, k\right) \in E$. Additionally, $\nexists v \in V \backslash\left\{u_{B_{i}}, S_{j}\right\}:(v, k) \in E$. Lastly, $\forall k \in B_{i}, \nexists v \in V:(k, v) \in E$, except $u_{B_{i}}$ that has edges only to $\left(H_{S_{j}}^{1} \cap I_{S_{i}}^{2}\right) \cup Y_{i}$ as defined previously.

Evidently, subset of $\left(H_{S_{j}}^{1} \cap I_{S_{i}}^{2}\right) \cup Y_{i}$ is the set $H_{S_{j}}^{1} \cap I_{S_{i}}^{2}$; therefore, area $B_{i}$ is constructed to give area $A_{j}$ the end that similarly area $A_{i}$ had (Figures 10,11 ).

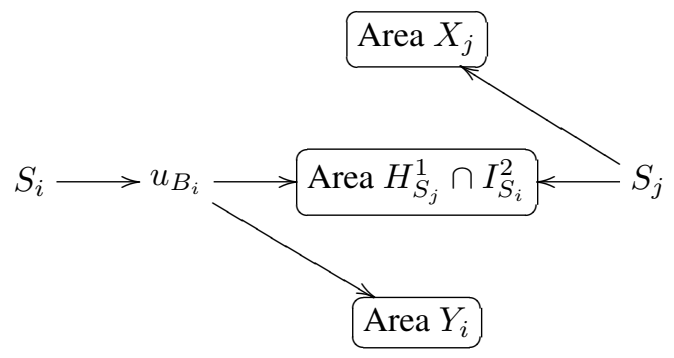

Figure 11: The partially shared area $B_{i} \cup A_{j}$ between the two strategies $\left(S_{i}, S_{j}\right) \in \mathcal{S}_{\mathcal{D}}{ }^{2}, S_{i} \neq S_{j}: B_{i} \equiv$ $\left(H_{S_{j}}^{1} \cap I_{S_{i}}^{2}\right) \cup Y_{i} \cup\left\{u_{B_{i}}\right\}$, and $A_{j} \equiv\left(H_{S_{j}}^{1} \cap I_{S_{i}}^{2}\right) \cup X_{j}$. Moreover, note that $\left|H_{S_{j}}^{1} \cap I_{S_{i}}^{2}\right|=\alpha_{S_{j} S_{i}}$, and $\left|Y_{i}\right|=\alpha_{\max }-\alpha_{S_{j} S_{i}}$. Therefore, $\left|B_{i}\right|=\alpha_{\max }+1$. Also, it is $\left|X_{j}\right|=\alpha_{\max }-\alpha_{S_{j} S_{i}}$, and as a result, $\left|A_{j}\right|=\alpha_{\max }$.

C. Name this part $C_{i}$ (Figure 12); through its structure we shall later show that the value of $\beta_{2}\left(S_{i}, S_{j}\right)$ can be independently decided from the values of $\alpha_{S_{i^{\prime}} S_{j^{\prime}}}, \beta_{2}\left(S_{i^{\prime}}, S_{j^{\prime}}\right), \forall\left(i^{\prime}, j^{\prime}\right) \in\{1, \ldots, r\}^{2}$. Assume, $C_{i} \subset H_{S_{i}}$, $\left|C_{i}\right|=\beta_{\text {max }}$, and that $\forall k \in C_{i},\left(S_{i}, k\right) \in E$, while $\nexists v \in V \backslash\left\{S_{i}, S_{j}\right\}:(v, k) \in E$. Additionally, assume $C_{i}=\left(H_{S_{i}}^{1} \cap H_{S_{j}}^{1}\right) \cup Z_{i}$, where $Z_{i} \equiv C_{i} \backslash\left(H_{S_{i}}^{1} \cap H_{S_{j}}^{1}\right)$, and that $\forall k \in Z_{i}, \nexists v \in V:(k, v) \in E$. Similarly, $\forall k \in H_{S_{i}}^{1} \cap H_{S_{j}}^{1}, \nexists v \in V:(k, v) \in E$. For simplicity we have assumed $I_{S_{i}}^{2} \cap I_{S_{j}}^{2}=\emptyset$, but the generalization is straightforward. Therefore, note that it is $\left|H_{S_{i}}^{1} \cap H_{S_{j}}^{1}\right|=\beta_{2}\left(S_{i}, S_{j}\right)$, i.e., $\left|Z_{i}\right|=\beta_{\max }-\beta_{2}\left(S_{i}, S_{j}\right)$.

Now, recall that we assumed $\beta_{2}\left(S_{i}, S_{j}\right)=\beta_{2}\left(S_{j}, S_{i}\right)$. Therefore, an area similar to $C_{i}$ exist also in $H_{S_{j}}$, i.e., an area $C_{j}$, that shares the same $H_{S_{i}}^{1} \cap H_{S_{j}}^{1}$ with $C_{i}$ (Figure 12).

Evidently, $H_{S_{j}}^{1} \cap I_{S_{i}}^{2}, H_{S_{j}}^{1} \cap H_{S_{i}}^{1}$, and $I_{S_{j}}^{2} \cap I_{S_{i}}^{2}$ can be independently decided from one another.

We can now justify our selection for $H$. Specifically, rewrite $H$ as $H=(r-1)\left(\alpha_{\max }+\left(1+\alpha_{\max }\right)+\beta_{\max }\right)+1+$ C: The factor $(r-1)$ is due to the fact that region $H_{S_{i}}$ is connected with - at most — the rest $(r-1)$ of the $H_{S_{(\cdot)}}$ regions (Figure 9). Furthermore, the first factor in the sum, $\alpha_{\max }$, is the cardinality of area $A_{i}$, while the second the cardinality of $B_{i}$, and the third the cardinality of $C_{i}$. Lastly, the unity corresponds to the node $S_{i}$, while C corresponds to the cardinality of a set $X_{i}$ such that $\forall k \in X_{i},\left(S_{i}, k\right) \in E$, while $\nexists v \in V \backslash\left\{S_{i}\right\}:(v, k) \in E$ and $\nexists v \in V:(k, v) \in E$. And as noted before, if c is sufficiently large, e.g. c $\geq \alpha_{\max }$, the set $\mathcal{S}_{\mathcal{D}}$ is a sink in $\mathcal{S}$. 


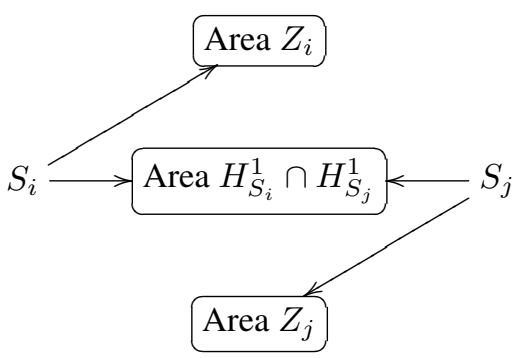

Figure 12: The partially shared area $C_{i} \cup C_{j}$ between the two strategies $\left(S_{i}, S_{j}\right) \in \mathcal{S}_{\mathcal{D}}{ }^{2}, S_{i} \neq S_{j}: C_{i}=$ $\left(H_{S_{i}}^{1} \cap H_{S_{j}}^{1}\right) \cup Z_{i}$, and $C_{j}=\left(H_{S_{i}}^{1} \cap H_{S_{j}}^{1}\right) \cup Z_{j}$. Moreover, note that here $\left|H_{S_{i}}^{1} \cap H_{S_{j}}^{1}\right|=\beta_{2}\left(S_{i}, S_{j}\right)$, and $\left|Z_{i}\right|=\left|Z_{j}\right|=\beta_{\max }-\beta_{2}\left(S_{i}, S_{j}\right)$. Therefore, $\left|C_{i}\right|=\left|C_{j}\right|=\beta_{\max }$.

We note that an obvious modification to the structure of $B_{i}$ can decrease $\mathrm{C}$ to zero (replace $u_{B_{i}}$ with a set $U_{B_{i}}$ of $\alpha_{\max }$ nodes such that $\forall u \in U_{B_{i}}$ it is $w_{S_{i} u}=\theta_{u}$, and $\forall v \in\left(H_{S_{j}}^{1} \cap I_{S_{i}}^{2}\right) \cup Y_{i}$ there exists a unique node $u \in U_{B_{i}}$ such that $w_{u v}=1$, while for any other node $u^{\prime} \neq u$ it is $\left.\left(u^{\prime}, v\right) \notin E\right)$.

As a final step to our proof, we prove that if

$$
\begin{aligned}
\text { i } \alpha_{\max } & =r-1 \text {, and } \\
\text { ii } \beta_{\max } & =\alpha_{\max }+r-1,
\end{aligned}
$$

then all the possible value orderings of $\left\{\alpha_{S_{i} S_{j}}\right\}_{(i, j) \in\{1, \ldots, r\}^{2}},\left\{\beta_{2}\left(S_{i}, S_{j}\right)\right\}_{(i, j) \in\{1, \ldots, r\}^{2}}$ are achievable.

First, we justify our selection for $\alpha_{\max }$. Recall that in our social network for $u_{1}^{i j} \geq u_{1}^{i^{\prime} j}$, where $i^{\prime} \in\{1, \ldots, r\} \backslash$ $\{i\}$ to hold, it must be $\alpha_{S_{j} S_{i}} \leq \alpha_{S_{j} S_{i^{\prime}}}$. Moreover, $\alpha_{S_{j} S_{i}} \in\{0,1, \ldots, r-1\}$, i.e., $\alpha_{S_{j} S_{i}}$ can take a value from a pool of $r$ different ones. On the other hand, $\alpha_{S_{j} S_{i}}$ is compared with exactly $(r-1)$ other $\alpha_{S_{j} S_{i^{\prime}}}$, since $i^{\prime} \in$ $\{1, \ldots, r\} \backslash\{i\}$. Thereby, there exist a set of surjective functions defined from the set $\left\{\alpha_{S_{i} S_{j}}\right\}_{(i, j) \in\{1, \ldots, r\}^{2}}$ to the set $\{0,1, \ldots, r-1\}$ that achieves all the possible value orderings of $\left\{\alpha_{S_{i} S_{j}}\right\}_{(i, j) \in\{1, \ldots, r\}^{2}}$ and, in accordance, all the possible value orderings of $u_{1}^{(\cdot) j}, \forall j \in\{1, \ldots, r\}$ (see also Remark 2 below).

Next, given that $\left\{\alpha_{S_{i} S_{j}}\right\}_{(i, j) \in\{1, \ldots, r\}^{2}}$ has been decided, we can validate our selection for $\beta_{\text {max }}$. First, recall that in our social network for $u_{2}^{i j} \geq u_{2}^{i j^{\prime}}$, where $j^{\prime} \in\{1, \ldots, r\} \backslash\{j\}$ such that $j \neq i$, and $j^{\prime} \neq i$, to hold, it must be $\beta_{2}\left(S_{i}, S_{j}\right) \leq \alpha_{S_{i} S_{j^{\prime}}}-\alpha_{S_{i} S_{j}}+\beta_{2}\left(S_{i}, S_{j^{\prime}}\right)$, (i.e., we do not consider the strategy profiles $(S, S)$, since $u_{2}(S, S)=0$, while $u_{2}\left(S_{i}, S_{j}\right) \geq 1, \forall S_{i}, S_{j} \in \mathcal{S}$, where $S_{i} \neq S_{j}$ - also, recall that $\gamma_{2}\left(S_{i}, S_{j}\right)=0$, $\forall\left(S_{i}, S_{j}\right) \in \mathcal{S}^{2}$, where $\left.S_{i} \neq S_{j}\right)$. Therefore, $\beta_{2}\left(S_{i}, S_{j}\right)$ is compared with exactly $(r-1)$ other $\beta_{2}\left(S_{i}, S_{j^{\prime}}\right)$, since $j^{\prime} \in\{1, \ldots, r\} \backslash\{j\}$. Now, we are able to give an algorithm that achieves all the possible value orderings of $\left\{\beta_{2}\left(S_{i}, S_{j}\right)\right\}_{(i, j) \in\{1, \ldots, r\}^{2}}$ and, in accordance, all the possible value orderings of $u_{2}^{i(\cdot)}, \forall i \in\{1, \ldots, r\}$, for any given $\left\{\alpha_{S_{i} S_{j}}\right\}_{(i, j) \in\{1, \ldots, r\}^{2}}$. Specifically,

1. For fixed $i \in\{1, \ldots, r\}, \forall\left(j, j^{\prime}\right) \in\{1, \ldots, r\}^{2}, j \neq j^{\prime}$ compute $\alpha_{S_{i} S_{j^{\prime}}}-\alpha_{S_{i} S_{j}}$.

2. Let $\beta=\max _{\left(j, j^{\prime}\right) \in\{1, \ldots, r\}^{2}}\left\{\alpha_{S_{i} S_{j^{\prime}}}-\alpha_{S_{i} S_{j}}\right\}$.

3. Define the set of surjective functions from the set $\left\{\beta_{2}\left(S_{i}, S_{j}\right)\right\}_{(i, j)}$, where $(i, j) \in\{1, \ldots, r\}^{2}$, to the set $\{\beta, \ldots, \beta+(r-1)\}$ that achieves all the possible value orderings of $\left\{\beta_{2}\left(S_{i}, S_{j}\right)\right\}_{(i, j) \in\{1, \ldots, r\}^{2}}$.

4. return this set of functions.

Since, $\beta \leq \alpha_{\max }$, and $\beta_{\max }=\alpha_{\max }+(r-1)$, the above algorithm works for all possible values of $\beta$, and the fact completes our proof.

Remark 2. We note with regard to the condition

$$
\alpha_{S_{j} S_{i}} \leq \alpha_{S_{j} S_{i^{\prime}}}, i^{\prime} \in\{1, \ldots, r\} \backslash\{i\},
$$


that $\forall x \in\{1, \ldots, r\}$ it is $a_{S_{x} S_{x}}=0$, i.e., $u_{1}\left(S_{x}, S_{x}\right)=H$, and as a result, $u_{1}\left(S_{x}, S_{x}\right)$ is always greater, or equal to any of the $u_{1}\left(S_{x^{\prime}}, S_{x}\right), x^{\prime} \in\{1, \ldots, r\} \backslash\{x\}$. On the other hand, this restriction can be nullified through simple perturbations to the constructed networks, by letting $\left|H_{S_{i}}\right|, \forall i \in\{1, \ldots, r\}$, differ slightly from $H$, and increasing appropriately the $\alpha_{\max }, \beta_{\max }$.

Proof of Corollary 1. We construct a certain class of graphs, denoted by $\mathcal{G}_{\mathcal{D}}^{\prime}$, that induce a class of social networks with the involved property. Specifically, our construction is based on the social networks of Theorem 2, from the proof of which we also inherent all the involved definitions and notations.

Specifically, we assume $r \geq 3$, and we first construct $r$ new disjoint graphs $R_{i}, i \in\{1, \ldots, r\}$, which we will append to the graph of Theorem 2:

i. For each $i \in\{1, \ldots, r\}$, construct a directed rooted tree $R_{i}=\left(V_{R_{i}}, E_{R_{i}}\right)$ such that $\forall i^{\prime} \in\{1, \ldots, r\}$ it is $\left|V_{R_{i}}\right|=\left|V_{R_{i^{\prime}}}\right|$, and $\left|E_{R_{i}}\right|=\left|V_{R_{i}}\right|-1$. Also, let $\rho_{R_{i}}$ denote its root. Thereby, at this point of the construction all nodes in $V_{R_{i}}$ have in-degree one, except of the root, and out-degree one, except of the leaves.

ii. Moreover, set $w_{u v}=\theta_{v}, \forall(u, v) \in E_{R_{i}}$.

iii. Now, add the edge $\left(S_{i}, \rho_{R_{i}}\right)$, and let $w_{S_{i} \rho_{R_{i}}}=\theta_{\rho_{R_{i}}}$. Therefore, all nodes in $V_{R_{i}}$ are reachable from $S_{i}$.

iv. Next, given all $R_{i}, \forall i \in\{1, \ldots, r\}$, add edges between the nodes in $V_{R_{i}}$, according to the $P_{\text {in }}$ and $P_{\text {out }}$ distributions, bearing in mind the preexisting structure of the graph of Theorem 2 , and with any weight with regard to the LTM.

v. Finally, due to the fact that all nodes in each $V_{R_{i}}$ have in and out-degree at least one, except of the leaves, we may have to add some further nodes according to the values of $P_{\text {in }}(0)$, and $P_{\text {out }}(0)$. We can add them such that $i$ ) they are not reachable from any other nodes, and $i$ ) they cannot reach any other nodes (e.g., by connecting them with any other nodes only with edges of insufficient weight with regard to the LTM). Similarly, for any further discrepancies between $P_{i n}$, and $P_{\text {out }}$ and the so far achieved distributions we can add further nodes in the same way.

Thus, we obtain a modified graph $G$ that has in-degree distribution $P_{i n}^{G}$ and out-degree distribution $P_{\text {out }}^{G}$ such that for all $i \geq 0$ it is $\left|P_{i n}(i)-P_{i n}^{G}(i)\right| /|V| \rightarrow 0$ as $|V| \rightarrow \infty$ (i.e., as $\left|V_{R_{i}}\right|, \forall i \in\{1, \ldots, r\}$, and the number of the extra nodes in case v increase), and the same holds for $P_{\text {out }}$ and $P_{\text {out }}^{G}$, since the preexisting structure, as in the proof of Theorem 2, is static given any fixed $r \geq 3$.

To complete our proof, we prove that the set of strategies $\mathcal{S}_{\mathcal{D}}$ is a sink in $\mathcal{S}$. To this end, let $\left(\lambda, \lambda^{\prime}\right) \in\{r+1, \ldots$, $|V|\}^{2}$, and $\left(i, j, j^{\prime}\right) \in\{1, \ldots, r\}^{3}$, where $i \neq j, i \neq j^{\prime}, j \neq j^{\prime}$, as well as, $S_{\lambda} \notin H_{S_{i}}, S_{\lambda} \notin H_{S_{j}}$, and $S_{\lambda^{\prime}} \notin H_{S_{j}}$. Next, consider the following part of the game matrix:

\begin{tabular}{c||c|c|c} 
& $S_{j}$ & $S_{j^{\prime}}$ & $S_{\lambda}$ \\
\hline \hline$S_{i}$ & $u_{2}^{i j}$ & $u_{2}^{i j^{\prime}}$ & $u_{2}^{i \lambda}$ \\
\hline$S_{j}$ & 0 & $u_{2}^{j j^{\prime}}$ & $u_{2}^{j \lambda}$ \\
\hline$S_{\lambda^{\prime}}$ & $u_{2}^{\lambda^{\prime} j}$ & $u_{2}^{\lambda^{\prime} j^{\prime}}$ & $u_{2}^{\lambda^{\prime} \lambda}$
\end{tabular}

We want,

i. $u_{2}^{i j}>u_{2}^{i \lambda}$ : Equivalently, we write $\alpha_{S_{i} S_{j}}+\beta_{2}\left(S_{i}, S_{j}\right)<\alpha_{S_{i} S_{\lambda}}+\beta_{2}\left(S_{i}, S_{\lambda}\right)+\gamma_{2}\left(S_{i}, S_{\lambda}\right)+\left|H_{S_{j}}\right|-\left|H_{S_{\lambda}}\right|$. However, $\alpha_{S_{i} S_{\lambda}}+\beta_{2}\left(S_{i}, S_{\lambda}\right)+\gamma_{2}\left(S_{i}, S_{\lambda}\right)=0$, since $S_{\lambda} \notin H_{S_{i}}$. Thus, we have $\alpha_{S_{i} S_{j}}+\beta_{2}\left(S_{i}, S_{j}\right)<$ $\left|H_{S_{j}}\right|-\left|H_{S_{\lambda}}\right|$. But, $\left|H_{S_{j}}\right|-\left|H_{S_{\lambda}}\right| \geq H=(r-1)\left(2 \alpha_{\max }+\beta_{\max }\right)+1+\mathrm{c}>\alpha_{\max }+\beta_{\max }=$ $\max _{(i, j) \in\{1, \ldots, r\}^{2}}\left\{\alpha_{S_{i} S_{j}}+\beta_{2}\left(S_{i}, S_{j}\right)\right\}$ (recall $H$ from the previous proof). Therefore, $u_{2}^{i j}>u_{2}^{i \lambda}$.

ii. $u_{2}^{j j^{\prime}}>u_{2}^{j \lambda}$ : Replace in the previous analysis $i$ with $j$, and $j$ with $j^{\prime}$.

iii. $u_{2}^{\lambda^{\prime} j}>u_{2}^{\lambda^{\prime} \lambda}$ : Equivalently we write $\alpha_{S_{\lambda^{\prime}} S_{j}}+\beta_{2}\left(S_{\lambda^{\prime}}, S_{j}\right)+\gamma_{2}\left(S_{\lambda^{\prime}}, S_{j}\right)<\alpha_{S_{\lambda^{\prime}} S_{\lambda}}+\beta_{2}\left(S_{\lambda^{\prime}}, S_{\lambda}\right)+$ $\gamma_{2}\left(S_{\lambda^{\prime}}, S_{\lambda}\right)+\left|H_{S_{j}}\right|-\left|H_{S_{\lambda}}\right|$. However, $\alpha_{S_{\lambda^{\prime}} S_{j}}+\beta_{2}\left(S_{\lambda^{\prime}}, S_{j}\right)+\gamma_{2}\left(S_{\lambda^{\prime}}, S_{j}\right)=0$, since $S_{\lambda^{\prime}} \notin H_{S_{j}}$. Thus, we have $\alpha_{S_{\lambda^{\prime}} S_{\lambda}}+\beta_{2}\left(S_{\lambda^{\prime}}, S_{\lambda}\right)+\gamma_{2}\left(S_{\lambda^{\prime}}, S_{\lambda}\right)+\left|H_{S_{j}}\right|-\left|H_{S_{\lambda}}\right|>0$, which always holds, since $\left|H_{S_{j}}\right|-\left|H_{S_{\lambda}}\right|>0$, and the proof is complete. 
Now, let $\mu \in\{r+1, \ldots,|V|\}$, while $(i, j) \in\{1, \ldots, r\}^{2}, i \neq j$. We can limit our analysis, from the Player's 1 perspective, to the following part of the game matrix:

\begin{tabular}{c||c|c|c|c|c} 
& $S_{1}$ & $\cdots$ & $S_{j}$ & $\cdots$ & $S_{r}$ \\
\hline \hline$S_{i}$ & $u_{1}^{i 1}$ & $\cdots$ & $u_{1}^{i j}$ & $\cdots$ & $u_{1}^{i r}$ \\
\hline$\vdots$ & $\vdots$ & $\ddots$ & $\vdots$ & $\ddots$ & $\vdots$ \\
\hline$S_{\mu}$ & $u_{1}^{\mu 1}$ & $\cdots$ & $u_{1}^{\mu j}$ & $\cdots$ & $u_{1}^{i r}$
\end{tabular}

It is $u_{1}^{\mu j} \leq\left|H_{S_{\mu}}\right|$. On the other hand, it is $u_{1}^{j j}=\left|H_{S_{j}}\right| \geq H+\left|H_{S_{\mu}}\right|>\left|H_{S_{\mu}}\right|$, since $\left(S_{j}, \rho_{R_{j}}\right) \in E$. Thereby, $u_{1}^{j j}>u_{1}^{\mu j}$. The fact completes our proof.

\section{Section 4.2: Proofs of Theorems 3, 4, Lemma 2, and Theorem 5.}

Proof of Theorem 3. For the following proof, given a strategy profile $\mathbf{s}=\left(S_{x}, S_{y}\right) \in \mathcal{S}^{2},(x, y) \in\{1, \ldots,|\mathcal{S}|\}^{2}$, we denote the players' utilities as $u_{i}^{x y}$, instead of $u_{i}\left(S_{x}, S_{y}\right), \forall i \in \mathcal{M}=\{1,2\}$.

In the following, we assume that $\mathcal{S}=\left\{S_{i}\right\}_{i \in\{1, \ldots,|\mathcal{S}|\}}$, where $\left|H_{S_{i}}\right| \geq\left|H_{S_{i+1}}\right|, \forall i \in\{1, \ldots,|\mathcal{S}|-1\}$.

We first consider a strategy profile $\mathbf{s}=\left(S_{i}, S_{j}\right) \in \mathcal{S}^{2}$, where $i>j$. Then, if $\left|H_{S_{i}}\right|<\left|H_{S_{j}}\right|$, s cannot be a PNE, since $u_{1}^{i j} \leq\left|H_{S_{i}}\right|<\left|H_{S_{j}}\right|=u_{1}^{j j}$. On the other hand, if $\left|H_{S_{i}}\right|=\left|H_{S_{j}}\right|$, for $\mathbf{s}$ to be a PNE, it must $u_{1}^{i j} \geq u_{1}^{i^{\prime} j}, \forall i^{\prime} \in\{1, \ldots,|\mathcal{S}|\}$. Thereby, by substituting in the previous inequality $u_{1}^{i j}=\left|H_{S_{i}}\right|$, and $u_{1}^{i^{\prime} j}=\left|H_{S_{i}^{\prime}}\right|-\alpha_{1}\left(S_{i}^{\prime}, S_{j}\right)-\gamma_{1}\left(S_{i}^{\prime}, S_{j}\right)$, we have $\left|H_{S_{i}}\right| \geq\left|H_{S_{i}^{\prime}}\right|-\alpha_{1}\left(S_{i}^{\prime}, S_{j}\right)-\gamma_{1}\left(S_{i}^{\prime}, S_{j}\right)$, which for $i^{\prime}=1$ gives $\left|H_{S_{i}}\right| \geq\left|H_{\max }\right|-\alpha_{1}\left(S_{1}, S_{j}\right)-\gamma_{1}\left(S_{1}, S_{j}\right)$. Moreover, it is $\alpha_{1}\left(S_{1}, S_{j}\right) \leq \min \left\{\left|H_{S_{j}}\right|,\left|H_{\max }\right|\right\}=\left|H_{S_{j}}\right|$, and as a result, from $\left|H_{S_{i}}\right| \geq\left|H_{\max }\right|-\alpha_{1}\left(S_{1}, S_{j}\right)-\gamma_{1}\left(S_{1}, S_{j}\right)$ we have $\left|H_{S_{i}}\right|+\left|H_{S_{j}}\right| \geq\left|H_{\max }\right|-\gamma_{1}\left(S_{1}, S_{j}\right)$. In other words, if $\mathbf{s}$ is a PNE, then it is $\left|H_{S_{i}}\right|+\left|H_{S_{j}}\right| \geq\left|H_{\text {max }}\right|-\gamma_{1}\left(S_{\text {max }}, S_{j}\right)$.

Now, we note that a strategy profile $\mathbf{s}=\left(S_{i}, S_{i}\right) \in \mathcal{S}^{2}$, cannot be a PNE, since $u_{2}^{i i}=0$, and even though, e.g., $\left|H_{S_{1}}\right|+\left|H_{S_{1}}\right|=2\left|H_{\max }\right|>\left|H_{\max }\right|$.

Next, given a strategy profile $\mathbf{s}=\left(S_{i}, S_{j}\right)$, we assume $i<j$. Then, for $\mathbf{s}$ to be a PNE, it must be $u_{1}^{i j} \geq u_{1}^{i^{\prime} j}, \forall i^{\prime} \neq$ $i,\left(i, i^{\prime}\right) \in\{1, \ldots,|\mathcal{S}|\}^{2}$. Thus, the inequality must also hold $\forall i^{\prime} \leq i$. Now, let $i^{\prime}=: i-x, x \in\{0, \ldots, i-1\}$, and substitute $u_{1}^{y j}=\left|H_{S_{y}}\right|-\alpha_{1}\left(S_{y}, S_{j}\right)-\gamma_{1}\left(S_{y}, S_{j}\right), y \in\{i, i-x\}$ to the previous inequality: We take $\alpha_{1}\left(S_{i}, S_{j}\right)+\gamma_{1}\left(S_{i}, S_{j}\right) \leq \alpha_{1}\left(S_{i-x}, S_{j}\right)+\gamma_{1}\left(S_{i-x}, S_{j}\right)+\left|H_{S_{i}}\right|-\left|H_{S_{i-x}}\right|, \forall x \in\{0, \ldots, i-1\}$. Thereby, we have $\alpha_{1}\left(S_{i-x}, S_{j}\right)+\gamma_{1}\left(S_{i-x}, S_{j}\right)+\left|H_{S_{i}}\right|-\left|H_{S_{i-x}}\right| \geq 0$. However, $\alpha_{1}\left(S_{i-x}, S_{j}\right) \leq \min \left\{\left|H_{S_{j}}\right|,\left|H_{S_{i-x}}\right|\right\}=$ $\left|H_{S_{j}}\right|$, due to our initial assumption and the fact that $i-x \leq i<j$. Thereby, it must hold $\left|H_{S_{j}}\right|+\left|H_{S_{i}}\right| \geq$ $\left|H_{S_{i-x}}\right|-\gamma_{1}\left(S_{i-x}, S_{j}\right), \forall x \in\{0, \ldots, i-1\}$, i.e., for $x=i-1$ must hold $\left|H_{S_{j}}\right|+\left|H_{S_{i}}\right| \geq\left|H_{S_{1}}\right|-\gamma_{1}\left(S_{1}, S_{j}\right)=$ $\left|H_{\max }\right|-\gamma_{1}\left(S_{\max }, S_{j}\right)$, and the proof is complete.

Proof of Theorem 4. Assume that $S_{1}$ is reachable from $S_{2}$, i.e., $S_{1} \subseteq H_{S_{2}}$. Then, $H_{S_{2}} \supseteq H_{S_{1}}$, and as a result, $u_{1}\left(S_{2}, S_{2}\right)=\left|H_{S_{2}}\right| \geq\left|H_{S_{1}}\right|>\left|H_{S_{1}}\right|-\left|S_{2} \backslash S_{1}\right| \geq u_{1}\left(S_{1}, S_{2}\right)$, since $S_{1} \neq S_{2}$, (i.e., since $S_{1} \neq S_{2}$, Player 1, by playing $S_{1}$, cannot color at least one of the nodes in $\left.S_{2}\right)$. Thereby, the strategy profile $\mathbf{s}=\left(S_{1}, S_{2}\right) \in \mathcal{S}^{2}$ is not a PNE.

At this point, note the trivial case where a single strategy $S$ exists in the game, i.e., $k=|V|$ (contrary to our basic assumption in this paper that $k<|V|)$ : Then, a unique trivial $\operatorname{PNE}(S, S)$ exists, where Player 1 has utility $\left|H_{S}\right|$, and Player 2 zero. On the other hand, $S$ is reachable by itself, since $S \subseteq H_{S}$ by definition.

Proof of Lemma 2. We treat only the first and the third case, since the proofs of the others are similar.

For the first case, let $\left(S_{1}, S_{2}\right) \in \mathcal{S}^{2}, S_{1} \neq S_{2}$, such that $S_{1}$ is reachable from $S_{2}$, and $S_{2}$ is reachable from $S_{1}$. Then, we deduce $H_{S_{1}}=H_{S_{2}}$. Consequently, it is also $\left|H_{S_{1}}\right|=\left|H_{S_{2}}\right|=: H$. Now, consider the following part of the game matrix: 


\begin{tabular}{c||c|c} 
& $S_{1}$ & $S_{2}$ \\
\hline \hline$S_{1}$ & $H, 0$ & $u_{1}\left(S_{1}, S_{2}\right), u_{2}\left(S_{1}, S_{2}\right)$ \\
\hline$S_{2}$ & $u_{1}\left(S_{2}, S_{1}\right), u_{2}\left(S_{2}, S_{1}\right)$ & $H, 0$
\end{tabular}

From Player's 1 perspective, it is $u_{1}\left(S_{1}, S_{2}\right)<\left|H_{S_{1}}\right|=H$, and similarly for $u_{1}\left(S_{2}, S_{1}\right)$. From Player's 2 perspective, it is $u_{2}\left(S_{1}, S_{2}\right)>0$ and, similarly for $u_{2}\left(S_{2}, S_{1}\right)$. Therefore, when $S_{1}$ is reachable from $S_{2}$, and $S_{2}$ is reachable from $S_{1}$ there is an improvement cycle. Thus, a generalized ordinal potential cannot exist.

For the third case, consider the following part of the game matrix:

\begin{tabular}{c||c|c} 
& $S_{1}$ & $S_{2}$ \\
\hline \hline$S_{1}$ & $\left|H_{S_{1}}\right|, 0$ & $u_{1}\left(S_{1}, S_{2}\right), u_{2}\left(S_{1}, S_{2}\right)$ \\
\hline$S_{2}$ & $u_{1}\left(S_{2}, S_{1}\right), u_{2}\left(S_{2}, S_{1}\right)$ & $\left|H_{S_{2}}\right|, 0$
\end{tabular}

It is $u_{1}\left(S_{2}, S_{1}\right) \leq\left|H_{S_{2}}\right|<\left|H_{S_{1}}\right|$. Thus, if $u_{1}\left(S_{1}, S_{2}\right)<\left|H_{S_{2}}\right|$ there is an improvement cycle. The fact completes our proof.

Proof of Theorem 5. First, we prove the following lemma.

Lemma 7. Consider a game $\Gamma=\left(\left(G, L I S, T B C 1=R^{\prec}, T B C 2\right), \mathcal{M}, k\right)$, and that $\left(S_{1}, S_{1}^{\prime}, S_{2}, S_{2}^{\prime}\right) \in \mathcal{S}^{4}$, for $S_{1} \neq S_{1}^{\prime}$ and $S_{2} \neq S_{2}^{\prime}$. If $\left|H_{S_{1}}\right| \geq\left|H_{S_{1}^{\prime}}\right|+\left|H_{S_{2}}\right|+\gamma_{1}\left(S_{1}, S_{2}\right)$, then $u_{1}\left(S_{1}, S_{2}\right) \geq u_{1}\left(S_{1}^{\prime}, S_{2}\right)$, and if $\left|H_{S_{2}}\right| \geq\left|H_{S_{2}^{\prime}}\right|+\left|H_{S_{1}}\right|+\gamma_{2}\left(S_{1}, S_{2}\right)$, then $u_{2}\left(S_{1}, S_{2}\right) \geq u_{2}\left(S_{1}, S_{2}^{\prime}\right)$.

Proof. For the following proof, given a strategy profile $\mathbf{s}=\left(S_{1}, S_{2}\right) \in \mathcal{S}$, we denote the players' utilities as $u_{i}^{12}$, instead of $u_{i}\left(S_{1}, S_{2}\right), \forall i \in \mathcal{M}$.

Let $S_{1} \neq S_{1}^{\prime}$, and consider the following part of the game matrix, from Player's 1 perspective:

\begin{tabular}{c||c} 
& $S_{2}$ \\
\hline \hline$S_{1}$ & $u_{1}^{12}$ \\
\hline$S_{1}^{\prime}$ & $u_{1}^{1^{\prime} 2}$
\end{tabular}

It is $u_{1}^{12} \in\left\{\max \left\{0,\left|H_{S_{1}}\right|-\left|H_{S_{2}}\right|-\gamma_{1}\left(S_{1}, S_{2}\right)\right\}, \ldots,\left|H_{S_{1}}\right|\right\}$. The lower bound is obtainable if Player 2, by playing $S_{2}$, colors the whole region $H_{S_{2}}$, even if Player 1 plays $S_{1}$. On the other hand, the upper bound is achievable when Player 1, by playing $S_{2}$, colors the whole region $H_{S_{1}}$, regardless the fact that Player 2 plays $S_{2}$. Similarly, it is $u_{1}^{1^{\prime} 2} \in\left\{\max \left\{0,\left|H_{S_{1}^{\prime}}\right|-\left|H_{S_{2}}\right|-\gamma_{1}\left(S_{1}^{\prime}, S_{2}\right)\right\}, \ldots,\left|H_{S_{1}^{\prime}}\right|\right\}$. Now, assume $\left|H_{S_{1}}\right|-\left|H_{S_{2}}\right|-$ $\gamma_{1}\left(S_{1}, S_{2}\right) \geq\left|H_{S_{1}^{\prime}}\right|$, i.e., $\left|H_{S_{1}}\right| \geq\left|H_{S_{1}^{\prime}}\right|+\left|H_{S_{2}}\right|+\gamma_{1}\left(S_{1}, S_{2}\right)$. Then, $u_{1}^{12} \geq u_{1}^{1^{\prime} 2}$.

Now, let $S_{2} \neq S_{2}^{\prime}$, and consider the following part of the game matrix, from Player's 2- perspective:

\begin{tabular}{c||c|c} 
& $S_{2}$ & $S_{2}^{\prime}$ \\
\hline \hline$S_{1}$ & $u_{2}^{12}$ & $u_{2}^{12^{\prime}}$
\end{tabular}

It is $u_{2}^{12} \in\left\{\max \left\{0,\left|H_{S_{2}}\right|-\left|H_{S_{1}}\right|-\gamma_{2}\left(S_{1}, S_{2}\right)\right\}, \ldots,\left|H_{S_{2}}\right|\right\}$. Similarly for $u_{2}^{12^{\prime}}$. Now, assume $\left|H_{S_{2}}\right|-$ $\left|H_{S_{1}}\right|-\gamma_{2}\left(S_{1}, S_{2}\right) \geq\left|H_{S_{2}^{\prime}}\right|$, i.e., $\left|H_{S_{2}}\right| \geq\left|H_{S_{2}^{\prime}}\right|+\left|H_{S_{1}}\right|+\gamma_{2}\left(S_{1}, S_{2}\right)$. Then, $u_{2}^{12} \geq u_{2}^{12^{\prime}}$, and the proof is complete.

For the rest of the proof, given a strategy profile $\mathbf{s}=\left(S_{x}, S_{y}\right) \in \mathcal{S}^{2},(x, y) \in\{1, \ldots,|\mathcal{S}|\}^{2}$, we denote the players' utilities as $u_{i}^{x y}$, instead of $u_{i}\left(S_{x}, S_{y}\right), \forall i \in \mathcal{M}$.

Moreover, we assume for simplicity that $\left|H_{S}\right|+\max \left\{\gamma_{1}(S, Y), \gamma_{2}(Y, S)\right\}$ is even, $\forall(S, Y) \in \mathcal{S}^{2}$.

Assume $\left(i, i^{\prime}, j\right) \in\{1, \ldots,|\mathcal{S}|\}^{3}$, and set $j>i^{\prime}>i$. We shall compare $u_{1}^{i j}$ and $u_{1}^{i^{\prime} j}$ : Based on the hypothesis, and the assumption that $j>i^{\prime}>i$, it is $\left|H_{S_{i}}\right| \geq 2\left(\left|H_{S_{i+1}}\right|+\gamma_{1}\left(S_{i}, S_{i+1}\right)\right)>\left|H_{S_{j}}\right|+\gamma_{1}\left(S_{i}, S_{j}\right)+\left|H_{S_{i^{\prime}}}\right|+$ $\gamma_{1}\left(S_{i}, S_{i}^{\prime}\right)>\left|H_{S_{j}}\right|+\left|H_{S_{i^{\prime}}}\right|+\gamma_{1}\left(S_{i}, S_{j}\right)$. Thereby, $u_{1}^{i j}>u_{1}^{i^{\prime} j}$, according to Lemma 7. 
Now, set $j=i^{\prime}>i$. We shall compare $u_{1}^{i j}$ and $u_{1}^{j j}$ : It is $\left|H_{S_{i}}\right| \geq 2\left(\left|H_{S_{i+1}}\right|+\gamma_{1}\left(S_{i}, S_{i+1}\right)\right) \geq\left|H_{S_{j}}\right|+\left|H_{S_{j}}\right|+$ $\gamma_{1}\left(S_{i}, S_{j}\right)$. Thus, $u_{1}^{i j} \geq u_{1}^{j j}$. However, it also is $u_{2}^{j j}=0$, therefore the strategy profile $\left(S_{j}, S_{j}\right)$ cannot be a PNE. In sum, Player 1 , from any strategy profile $\left(S_{i^{\prime}}, S_{j}\right)$ such that $i^{\prime}<j$, can deviate to any strategy profile $\left(S_{i}, S_{j}\right)$, $i<i^{\prime}$, and increase his utility.

Similarly, we can prove that Player 2 , from any strategy profile $\left(S_{i}, S_{j^{\prime}}\right)$ such that $j^{\prime} \leq i$, can deviate to any strategy profile $\left(S_{i}, S_{j}\right), j<j^{\prime}$, and increase his utility.

Now, due to the previous discussion no improvement cycles exist — i.e., a generalized ordinal potential exists [25, 32 ] - and the candidate sets of strategy profiles, where a PNE can exist, are the

i. $\left(S_{1}, S_{j}\right), \forall j \in\{2, \ldots,|\mathcal{S}|\}$, (for $j=1$ it is $u_{2}^{11}=0$ ), and

ii. $\left(S_{i}, S_{1}\right), \forall i \in\{1, \ldots,|\mathcal{S}|\}$. However, $u_{1}^{11}=\left|H_{S_{1}}\right|>u_{1}^{i 1}, \forall i \in\{2, \ldots,|\mathcal{S}|\}$, since $u_{1}^{i 1} \leq\left|H_{S_{i}}\right|$, and $\left|H_{S_{i}}\right|<\left|H_{S_{1}}\right|$ from the hypothesis.

Thereby, all PNE have the form $\left(S_{\max }, S_{j}\right)$, and our proof is complete.

\section{E Section 4.3: Proofs of Theorems 6, 7.}

Proof of Theorem 6. Firstly, consider the $S_{1}, S_{1}^{\prime}, S_{2}, S_{2}^{\prime}$, where $S_{1} \neq S_{1}^{\prime}$, and $S_{2} \neq S_{2}^{\prime}$, and set $\mathbf{s}=\left(S_{1}, S_{2}\right)$. Now, let Player 1 diverge from $S_{1}$ to a better strategy $S_{1}^{\prime}$. Specifically, set $\mathbf{s}^{\prime}=\left(S_{1}^{\prime}, S_{2}\right)$, and let $K_{1} \geq k+1$ be the increasing step, such that

$$
\begin{aligned}
u_{1}\left(\mathbf{s}^{\prime}\right) & =u_{1}(\mathbf{s})+K_{1} \Leftrightarrow \\
\left|H_{S_{1}^{\prime}}\right|-\alpha_{1}\left(\mathbf{s}^{\prime}\right) & =\left|H_{S_{1}}\right|-\alpha_{1}(\mathbf{s})+K_{1} \Rightarrow \\
\left|H_{S_{1}^{\prime}}\right| & \geq\left|H_{S_{1}}\right|+1,
\end{aligned}
$$

where the first equivalence holds because $D=1$, (i.e., $\gamma_{1}(\mathbf{s})=0, \forall \mathbf{s} \in \mathcal{S}^{2}$ ), while the last inequality holds since $\left|H_{S_{1}^{\prime}}\right|-\alpha_{1}\left(\mathbf{s}^{\prime}\right) \leq\left|H_{S_{1}^{\prime}}\right|$, and $K_{1}-\alpha_{1}(\mathbf{s}) \geq 1$ (for $D=1$ it is $\left.\alpha_{1}(\mathbf{s}) \leq k\right)$. Moreover, $\beta_{2}\left(\mathbf{s}^{\prime}\right)+\gamma_{2}\left(\mathbf{s}^{\prime}\right) \leq$ $\beta_{\max }+\gamma_{\max }+\beta_{2}(\mathbf{s})+\gamma_{2}(\mathbf{s})$. Therefore, after substitution we get:

$$
\begin{aligned}
P\left(\mathbf{s}^{\prime}\right)= & \left(1+\beta_{\max }+\gamma_{\max }\right)\left|H_{S_{1}^{\prime}}\right|+\left|H_{S_{2}}\right|-\beta_{2}\left(\mathbf{s}^{\prime}\right)-\gamma_{2}\left(\mathbf{s}^{\prime}\right) \\
\geq & \left(1+\beta_{\max }+\gamma_{\max }\right)\left|H_{S_{1}}\right|+1+\beta_{\max }+\gamma_{\max }+\left|H_{S_{2}}\right|-\beta_{2}(\mathbf{s})-\gamma_{2}(\mathbf{s}) \\
& -\beta_{\max }-\gamma_{\max } \\
> & P(\mathbf{s}) .
\end{aligned}
$$

Now, let Player 2 diverge from $S_{2}$ to a better strategy $S_{2}^{\prime}$. Specifically, set $\mathbf{s}^{\prime}=\left(S_{1}, S_{2}^{\prime}\right)$, and let $K_{2} \geq k+1$ be the increasing step, so that

$$
\begin{aligned}
u_{2}\left(\mathbf{s}^{\prime}\right) & =u_{2}(\mathbf{s})+K_{2} \Leftrightarrow \\
\left|H_{S_{2}^{\prime}}\right|-\alpha_{2}\left(\mathbf{s}^{\prime}\right)-\beta_{2}\left(\mathbf{s}^{\prime}\right)-\gamma_{2}\left(\mathbf{s}^{\prime}\right) & =\left|H_{S_{2}}\right|-\alpha_{2}(\mathbf{s})-\beta_{2}(\mathbf{s})-\gamma_{2}(\mathbf{s})+K_{2} \Rightarrow \\
\left|H_{S_{2}^{\prime}}\right|-\beta_{2}\left(\mathbf{s}^{\prime}\right)-\gamma_{2}\left(\mathbf{s}^{\prime}\right) & \geq\left|H_{S_{2}}\right|-\beta_{2}(\mathbf{s})-\gamma_{2}(\mathbf{s})+1,
\end{aligned}
$$

since $K_{2}-\alpha_{2}(\mathbf{s}) \geq 1$ (for $D=1$ it is $\alpha_{2}(\mathbf{s}) \leq k$ ). Therefore,

$$
\begin{aligned}
P\left(\mathbf{s}^{\prime}\right) & =\left(1+\beta_{\max }+\gamma_{\max }\right)\left|H_{S_{1}}\right|+\left|H_{S_{2}^{\prime}}\right|-\beta_{2}\left(\mathbf{s}^{\prime}\right)-\gamma_{2}\left(\mathbf{s}^{\prime}\right) \\
& \geq\left(1+\beta_{\max }+\gamma_{\max }\right)\left|H_{S_{1}}\right|+\left|H_{S_{2}}\right|-\beta_{2}(\mathbf{s})-\gamma_{2}(\mathbf{s})+1 \\
& >P(\mathbf{s}) . \quad \square
\end{aligned}
$$

Example 6 (Approximation $\epsilon=\mathbf{k}$ in Theorem 6 is tight). Let the game $\left(\left(G, L I S=L T M, T B C 1=R^{\prec}, T B C 2=\right.\right.$ $\left.\left.R^{\prec}\right), \mathcal{M}, k\right)$, where $D=1$, over the social network in Figure 13, where we have assumed without loss of generality that $|V|=2$, and $k=1$ (generally, we could consider a clique of $|V|=2 k$ nodes, where $k \geq 1$, and such that $w_{u v}=\theta_{v}, \forall(u, v) \in E$, with regard to the LTM). Then, the induced game matrix is as in Table 4; evidently, there is no PNE; nevertheless, the game has a 1-generalized ordinal potential. 


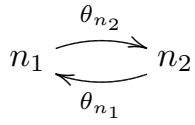

Figure 13: Tight example for Theorem 6.

\begin{tabular}{c||c|c} 
& $n_{1}$ & $n_{2}$ \\
\hline \hline$n_{1}$ & 2,0 & 1,1 \\
\hline$n_{2}$ & 1,1 & 2,0
\end{tabular}

Table 4: The game matrix for the game over the network in Figure 13.

Proof of Theorem 7. Firstly, assume $\left(S_{1}, S_{1}^{\prime}, S_{2}, S_{2}^{\prime}\right) \in \mathcal{S}^{4}$, where $S_{1} \neq S_{1}^{\prime}$, and $S_{2} \neq S_{2}^{\prime}$, and set $\mathbf{s}=\left(S_{1}, S_{2}\right)$. Now, let Player 1 diverge from $S_{1}$ to a better strategy $S_{1}^{\prime}$. Specifically, set $\mathbf{s}^{\prime}=\left(S_{1}^{\prime}, S_{2}\right)$, and let $K_{1} \geq D C_{\max }+1$ such that

$$
\begin{aligned}
u_{1}\left(\mathbf{s}^{\prime}\right) & =u_{1}(\mathbf{s})+K_{1} \Leftrightarrow \\
\left|H_{S_{1}^{\prime}}\right| & =\left|H_{S_{1}}\right|+\left(\alpha_{1}\left(\mathbf{s}^{\prime}\right)+\gamma_{1}\left(\mathbf{s}^{\prime}\right)\right)-\left(\alpha_{1}(\mathbf{s})+\gamma_{1}(\mathbf{s})\right)+K_{1} \Rightarrow \\
\left|H_{S_{1}^{\prime}}\right| & \geq\left|H_{S_{1}}\right|+1,
\end{aligned}
$$

since $K_{1} \geq D C_{\max }+1$. Moreover, $\beta_{2}\left(\mathbf{s}^{\prime}\right) \leq \beta_{\max }+\beta_{2}(\mathbf{s})$. Therefore,

$$
\begin{aligned}
P\left(\mathbf{s}^{\prime}\right) & =x_{1}\left|H_{S_{1}^{\prime}}\right|+\left|H_{S_{2}}\right|-\beta_{2}\left(\mathbf{s}^{\prime}\right) \\
& \geq x_{1}\left|H_{S_{1}}\right|+x_{1}+\left|H_{S_{2}}\right|-\beta_{2}(\mathbf{s})-\beta_{\max } \\
& >P(\mathbf{s}) .
\end{aligned}
$$

Now, let Player 2 diverge from $S_{2}$ to a better strategy $S_{2}^{\prime}$. Specifically, set s' $=\left(S_{1}, S_{2}^{\prime}\right)$, and let $K_{2} \geq D C_{\max }+1$ such that

$$
\begin{aligned}
u_{2}\left(\mathbf{s}^{\prime}\right) & =u_{2}\left(\mathbf{s}^{\prime}\right)+K_{2} \Leftrightarrow \\
\left|H_{S_{2}^{\prime}}\right|-\alpha_{2}\left(\mathbf{s}^{\prime}\right)-\beta_{2}\left(\mathbf{s}^{\prime}\right)-\gamma_{2}\left(\mathbf{s}^{\prime}\right) & =\left|H_{S_{2}}\right|-\alpha_{2}(\mathbf{s})-\beta_{2}(\mathbf{s})-\gamma_{2}(\mathbf{s})+K_{2} \Rightarrow \\
\left|H_{S_{2}^{\prime}}\right|-\beta_{2}\left(\mathbf{s}^{\prime}\right) & \geq\left|H_{S_{2}}\right|-\beta_{2}(\mathbf{s})+1,
\end{aligned}
$$

since $K_{2} \geq D C_{\max }+1$. Therefore,

$$
\begin{aligned}
P\left(\mathbf{s}^{\prime}\right) & =x_{1}\left|H_{S_{1}}\right|+\left|H_{S_{2}^{\prime}}\right|-\beta_{2}\left(\mathbf{s}^{\prime}\right) \\
& \geq x_{1}\left|H_{S_{1}}\right|+\left|H_{S_{2}}\right|-\beta_{2}(\mathbf{s})+1 \\
& >P(\mathbf{s}) . \quad \square
\end{aligned}
$$

Example 7 (Approximation $\mathbf{D C}_{\max }$ in Theorem 7 is tight). Let the game $((G, L I S=L T M, T B C 1=$ $\left.\left.R^{\prec}, T B C 2=R^{\prec}\right), \mathcal{M}, k\right)$, such that $D=2$, over the social network in Figure 14, where we have assumed without loss of generality that $|V|=3$, and $k=1$ (for the description of the general case, see below). Then, the induced game matrix is as in Table 5; evidently, there is no PNE; nevertheless, the game has an $\epsilon$-generalized ordinal potential only if $\epsilon=D C_{\max }=2$ (for $\mathbf{s}=\left(n_{2}, n_{2}\right)$, and $\mathbf{s}^{\prime}=\left(n_{1}, n_{2}\right)$, it is $D C_{\max }=D C_{1}\left(n_{1}, n_{2} \mid n_{2}\right)=$ $\left(\alpha_{1}\left(\mathbf{s}^{\prime}\right)+\gamma_{1}\left(\mathbf{s}^{\prime}\right)\right)-\left(\alpha_{1}(\mathbf{s})+\gamma_{1}(\mathbf{s})\right)=2$, since it is $\alpha_{1}\left(\mathbf{s}^{\prime}\right)=2$, and $\left.\alpha_{1}(\mathbf{s})=\gamma_{1}\left(\mathbf{s}^{\prime}\right)=\gamma_{1}(\mathbf{s})=0\right)$.

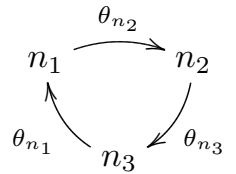

Figure 14: Tight example for Theorem 7.

\begin{tabular}{c||c|c|c} 
& $n_{1}$ & $n_{2}$ & $n_{3}$ \\
\hline \hline$n_{1}$ & 3,0 & 1,2 & 2,1 \\
\hline$n_{2}$ & 2,1 & 3,0 & 1,2 \\
\hline$n_{3}$ & 1,2 & 2,1 & 3,0
\end{tabular}

Table 5: The game matrix for the game over the network in Figure 14.

Generally, we could consider a social network similar to the one in Figure 14, where the nodes $n_{1}, n_{2}$, and $n_{3}$ are replaced by the sets of $k$ nodes $S_{1}, S_{2}$, and $S_{3}$, respectively, and the edges $\left(n_{1}, n_{2}\right),\left(n_{2}, n_{3}\right)$, and $\left(n_{3}, n_{1}\right)$, with the following: For $i \in\{1,2,3\}$, and $j=(i \bmod 3)+1$, we consider $\forall v_{i} \in S_{i}, \forall v_{j} \in S_{j}$, that $w_{v_{i} v_{j}}=\theta_{v_{j}} / k-$ i.e., only if all the nodes in $S_{i}$ are colored by a unique color, they can infect any node in $S_{j}$. Moreover, no other edges exist in the network. 


\section{F Section 4.4: Underlying network structure, and proof of Theorem 8.}

\section{F.1 Special Case $D(\Gamma)=k=1$ : Underlying network structure}

We restrict our analysis over a weakly connected component of $G$. Evidently, because the diffusion diameter, as well as, the parameter $k$ are equal to one, we assume $\operatorname{LTM}\left(w_{v u} \geq \theta_{u}, \forall(v, u) \in E\right)$, without loss of generality.

Next, we consider a weakly connected component (WCC) $\Delta$ of graph $G$, and some node $v \in V$ such that $d_{v}^{\text {out }} \geq 1$. Then, we distinguish between the following:

1. No other node $u \in \Delta$ has $d_{u}^{\text {out }} \geq 1$ (Figure 15a): As a result, the component is like a star with center node the node $v: \forall u \in \Delta$, where $u \neq v$, it is $(v, u) \in E$.

2. There exists at least one additional node $u \in \Delta, u \neq v$, that has $d_{u}^{\text {out }} \geq 1$ : We separate further our analysis: Specifically, for each such pair of nodes $u, v$ we consider the connections between $u, v$ and their out-neighbors, i.e., the nodes that they influence:

i. $(v, u) \notin E$, and $(u, v) \notin E$ (Figure 15b). Therefore, the component has at least two stars - one for each node with out-degree more than one. Moreover, due to the connectivity of $\Delta$, these stars must share with each other at least one leaf: For example, in Figure $15 \mathrm{~b}$ the node $n_{5}$ is a shared leaf.

ii. $(v, u) \in E$, whereas $(u, v) \notin E$ (Figure $15 \mathrm{c}$ ). Now, since $D=1$, node $v$ must have out-going edges to all the nodes that $u$ also has.

Note that $\left|H_{u} \backslash\{u\}\right| \geq 1$, since $u$ has at least one out-going edge; therefore, $\left|H_{v} \backslash\{v\}\right| \geq 2$, since $v$ points not only to $u$, but also to each node that $u$ does. Generally, $H_{u} \backslash\{u\} \subseteq H_{v}$, i.e., $\left|H_{u}\right| \geq\left|H_{v}\right|+1$, since $v \in H_{v}$ by definition.

iii. $(v, u) \notin E$, whereas $(u, v) \in E$. This case is symmetric to the previous one.

iv. $(v, u) \in E$, and $(u, v) \in E$ (Figure 15d). By combining the two previous cases, it is $\left|H_{u}\right|=\left|H_{v}\right|$.

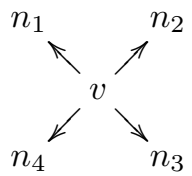

(a) A WCC for case 1 .

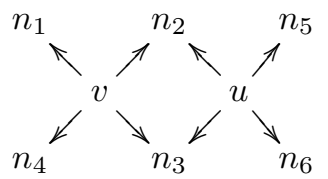

(b) A WCC for case $2 \mathrm{i}$.

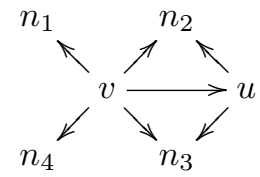

(c) A WCC for case 2 ii.

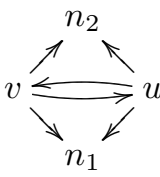

(d) A WCC for case 2iv.

Figure 15: The possible underlying weakly connected components (WCC) of a social network $\mathscr{N}$, given a game $(\mathscr{N}, \mathcal{M}, k=1)$, where $D=1$ (only WCCs with at most two nodes $v, u$ such that $d_{v}^{\text {out }}, d_{u}^{\text {out }} \geq 1$ are depicted).

\section{G Proof of Theorem 8.}

Proof of Theorem 8. We assume that the players' set of available strategies constitutes from all the nodes $v \in V$ such that $d_{v}^{\text {out }} \geq 1$, given that at least two of them exist, (otherwise, only trivial PNE exist, where Player 1 initiates the node $v \in V$ such that $d_{v}^{\text {out }} \geq 1$, and Player 2 initiates any of the remaining ones). Therefore, instead of $(v, u) \in E$, we shall write $\left(S_{v}, u\right)$ to denote the out-going edge from a node $v$, that has $d_{v}^{\text {out }} \geq 1$, to a node $u \in V, u \neq v$.

In the following, we assume that the strategy $S_{v} \in V$ - associated with node $v \in V$ - achieves the maximum ideal spread $\left|H_{\max }\right|$.

1. Assume that $S_{i} \notin H_{S_{j}} \backslash\left\{S_{j}\right\}, \forall(i, j) \in V^{2}$ such that $j \neq i$ : Then, according to the following lemma, the game $\Gamma$ admits a generalized ordinal potential. 
Lemma 8. A game $\Gamma=\left(\left(G, L I S=L T M, T B C 1=R^{\prec}, T B C 2=R^{\prec}\right), \mathcal{M}, k=1\right)$, where $D(\Gamma)=1$, and $S_{v} \notin H_{S_{u}} \backslash\left\{S_{u}\right\}, \forall(v, u) \in V^{2}$ with $u \neq v$, admits the generalized ordinal potential $P(\mathbf{s})=(1+$ $|V|)\left|H_{S_{1}}\right|+\left|H_{S_{2}}\right|-\left|H_{S_{1}} \cap H_{S_{2}}\right|, \forall \mathbf{s}=\left(S_{1}, S_{2}\right) \in \mathcal{S}^{2}$. Moreover, all its PNEs have the form $\left(S_{\text {max }}, S_{2}\right)$, where $S_{\text {max }} \equiv \operatorname{argmax}_{S \in \mathcal{S}}\left\{\left|H_{S}\right|\right\}$.

Proof. Recall that the players' set of available strategies constitutes from all the nodes $v \in V$ such that $d_{v}^{\text {out }} \geq 1$, given that at least two of them exist, (otherwise, only trivial PNE exist, where Player 1 initiates the node $v \in V$ such that $d_{v}^{\text {out }} \geq 1$, and Player 2 initiates any of the remaining ones).

Next, observe that under the hypothesis that $S_{v} \notin H_{S_{u}} \backslash\left\{S_{u}\right\}, \forall(v, u) \in V^{2}$ where $u \neq v$, the player's utilities (Lemma 1) can be written as follows, given a strategy profile $\mathbf{s} \in \mathcal{S}^{2}$ :

$$
\begin{aligned}
& u_{1}(\mathbf{s})=\left|H_{S_{1}}\right| \\
& u_{2}(\mathbf{s})=\left|H_{S_{2}}\right|-\left|H_{S_{1}} \cap H_{S_{2}^{\prime}}\right|
\end{aligned}
$$

Now, assume $\left(S_{1}, S_{1}^{\prime}, S_{2}, S_{2}^{\prime}\right) \in \mathcal{S}^{4}$, where $S_{1} \neq S_{1}^{\prime}$, and $S_{2} \neq S_{2}^{\prime}$. Now, let Player 1 diverge from $S_{1}$ to a better strategy $S_{1}^{\prime}$. Specifically, set $\mathbf{s}^{\prime}=\left(S_{1}^{\prime}, S_{2}\right)$, and let $K_{1} \geq 1$ be the increasing step such that

$$
\begin{aligned}
u_{1}\left(\mathbf{s}^{\prime}\right) & =u_{1}(\mathbf{s})+K_{1} \Leftrightarrow \\
\left|H_{S_{1}^{\prime}}\right| & =\left|H_{S_{1}}\right|+K_{1} \Rightarrow \\
\left|H_{S_{1}^{\prime}}\right| & \geq\left|H_{S_{1}}\right|+1 .
\end{aligned}
$$

In other words, whenever Player 1 digresses to a better strategy $S_{1}^{\prime}$, it increases its spread $\left|H_{S_{1}^{\prime}}\right|$. Moreover, $\left|H_{S_{1}^{\prime}} \cap H_{S_{2}}\right| \leq\left|H_{S_{1}} \cap H_{S_{2}}\right|+|V|$. Therefore,

$$
\begin{aligned}
P\left(\mathbf{s}^{\prime}\right) & =(1+|V|)\left|H_{S_{1}^{\prime}}\right|+\left|H_{S_{2}}\right|-\left|H_{S_{1}^{\prime}} \cap H_{S_{2}}\right| \\
& \geq(1+|V|)\left|H_{S_{1}}\right|+1+|V|+\left|H_{S_{2}}\right|-\left|H_{S_{1}} \cap H_{S_{2}}\right|-|V| \\
& >P(\mathbf{s}) .
\end{aligned}
$$

Now, let Player 2 diverge from $S_{2}$ to a better strategy $S_{2}^{\prime}$. Specifically, set $\mathbf{s}^{\prime}=\left(S_{1}, S_{2}^{\prime}\right)$, and let $K_{2} \geq 1$ be the increasing step such that

$$
\begin{aligned}
u_{2}\left(\mathbf{s}^{\prime}\right) & =u_{2}(\mathbf{s})+K_{2} \Leftrightarrow \\
\left|H_{S_{2}^{\prime}}\right|-\left|H_{S_{1}} \cap H_{S_{2}^{\prime}}\right| & =\left|H_{S_{2}}\right|-\left|H_{S_{1}} \cap H_{S_{2}}\right|+K_{2} \Rightarrow \\
\left|H_{S_{2}^{\prime}}\right|-\left|H_{S_{1}} \cap H_{S_{2}^{\prime}}\right| & \geq\left|H_{S_{2}}\right|-\left|H_{S_{1}} \cap H_{S_{2}}\right|+1 .
\end{aligned}
$$

Moreover, $\left|H_{S_{1}^{\prime}} \cap H_{S_{2}}\right| \leq\left|H_{S_{1}} \cap H_{S_{2}}\right|+|V|$. Therefore,

$$
\begin{aligned}
P\left(\mathbf{s}^{\prime}\right) & =(1+|V|)\left|H_{S_{1}}\right|+\left|H_{S_{2}^{\prime}}\right|-\left|H_{S_{1}} \cap H_{S_{2}^{\prime}}\right| \\
& \geq(1+|V|)\left|H_{S_{1}}\right|+\left|H_{S_{2}}\right|-\left|H_{S_{1}} \cap H_{S_{2}}\right|+1 \\
& >P(\mathbf{s}) . \quad \square
\end{aligned}
$$

2. Assume that exists at least one pair $\left(S_{v}, S_{u}\right) \in V^{2}$ such that $\left(S_{v}, S_{u}\right) \in E$, and $\left(S_{u}, S_{v}\right) \notin E$ : Then $\nexists k \in V$ such that $\left(S_{k}, S_{v}\right) \in E$ if also $\left(S_{v}, S_{k}\right) \notin E$, since that would imply $\left|H_{S_{k}}\right|>\left|H_{S_{v}}\right|=\left|H_{\text {max }}\right|$ (recall that the assumption $D=1$ implies that $k$ connects to all the out-neighbors of $v$ if $\left(S_{k}, S_{v}\right) \in E$ ), which contradicts our assumption that $\left|H_{S_{v}}\right|=\left|H_{\max }\right|$. We have the following further cases:

(a) Assume that $\exists k \in V,\left(S_{v}, S_{k}\right) \in E$, and $\left(S_{k}, S_{v}\right) \notin E$ : Then $\left|H_{S_{v}}\right|>\left|H_{S_{k}}\right|$. Assume that Player 2 plays $S_{k}$, and let Player 1 play $S_{v}$. Then, Player 1 shall have utility $u_{1}\left(S_{v}, S_{k}\right)=\left|H_{\max }\right|-1$, and Player 2 a utility $u_{2}\left(S_{v}, S_{k}\right)=1$. Now, if Player 2 does not have a better strategy to diverge to, then the strategy profile $\left(S_{v}, S_{k}\right)$ is a PNE.

On the other hand, if Player 2 has an alternative $S$ to play, then he will always obtain a utility greater, or equal to one, while Player 1 will obtain, by playing $S_{v}$, a utility $u_{1}\left(S_{v}, S\right)=\left|H_{\max }\right|$, if $S \notin H_{S_{v}}$, or $u_{1}\left(S_{v}, S\right)=\left|H_{\max }\right|-1$, if $S \in H_{S_{v}}$. Particularly, Player 2 can obtain a higher utility only if there is a 
strategy $S$ such that $\left(S_{v}, S\right) \notin E$, which means that the utility of Player 1 will then be $\left|H_{\max }\right|$, and that the strategy profile $\left(S_{v}, S\right)$ is a PNE.

Next, assume that Player 2 prefers to play $S_{k}$, given that Player 1 plays $S_{v}$, and that Player 1 has an alternative strategy $S_{v}^{\prime} \neq S_{v}$, that gives him utility $u_{1}\left(S_{v}^{\prime}, S_{k}\right)=\left|H_{\max }\right|$. However, that means that $k \notin H_{S_{v}^{\prime}}$, or else it would be $\left|H_{S_{v}^{\prime}}\right|=\left|H_{\max }\right|+1$ : contradiction. Therefore, there exists a node $b \in H_{S_{v}^{\prime}}$, that it is not in $H_{S_{v}}$, such that $u_{1}\left(S_{v}^{\prime}, S_{k}\right)$ can be equal to $\left|H_{\max }\right|$. Next observe that $v \notin H_{S_{v}^{\prime}}$, or else it must be $k \in H_{S_{v}}$, due to the fact that $D=1$. As a result, given that Player 1 plays strategy $S_{v}$ (and not the alternative $S_{v}^{\prime}$ ), if Player 2 picks $S_{v}^{\prime}$, then Player 1 obtains a utility $\left|H_{\max }\right|$, and Player 2 a utility of at least 2 , since he infects the nodes $v^{\prime}$, and $b$ : a contradiction. Hence, the strategy profile $\left(S_{v}, S_{k}\right)$ is a PNE.

(b) Assume $\left(S_{v}, S_{k}\right) \in E$, and $\left(S_{k}, S_{v}\right) \in E$. Then, it is also $\left|H_{S_{k}}\right|=\left|H_{\max }\right|$. The proof is similar to the previous case.

\section{H Section 5.1: Proof of Theorem 9.}

Proof of Theorem 9i. Case 1: We construct a social network such that the induced game over it has price of anarchy $|V| /(m k)$, and price of stability 1 . Specifically, we consider the left social network of Figure 4: As $V$ we assume the union of following sets of nodes: $X_{0} \cup X_{1} \cup \ldots \cup X_{m-1} \cup X_{r}$, where $\left|X_{0}\right|=\left|X_{1}\right|=\ldots=$ $\left|X_{m-1}\right|=k$, and $\left|X_{r}\right|$ can be arbitrarily large. Moreover, as $E$ we assume the union of following sets of edges:

i. $\forall u \in X_{0}, \forall v \in X_{1} \cup \ldots \cup X_{m-1}$ there is an edge $(u, v)$ with $w_{u v}^{\prime}=1 / k$.

ii. $\forall u \in X_{1} \cup \ldots \cup X_{m-1}, \forall v \in X_{r}$ there is an edge $(u, v)$ with $w_{u v}^{\prime}=1 /[(m-1) k]$.

Furthermore, in Figure 4, the edges $\left(X_{0}, X_{i}\right)$, where $i \in\{1, \ldots, m-1\}$, are annotated with the accumulated corresponding weight of the underlying edges between each of the nodes in $X_{0}$ and each of the nodes in $X_{i}$, and with regard to the LTM. In other words, $X_{0}$ accumulatively points with weight 1 to each node in $X_{i}$. Similarly, for the edges $\left(X_{i}, X_{r}\right)$.

Additionally, all nodes in $V$ are assumed to have threshold 1.

Thereby, only if the entire set $X_{0}$ is uniquely colored by a single firm it can color all the nodes in each of the $X_{i}, i \in\{1, \ldots, m-1\}$. On the other hand, $X_{0}$ cannot color any other node in $V$. Additionally, only if the entire $X_{1} \cup \ldots \cup X_{m-1}$ area is uniquely colored it can color $X_{r}$. Nevertheless, it cannot color any other subset of the graph.

Furthermore, the maximal social welfare of the induced game is exactly $|V|$. Specifically, given $i \in \mathcal{M}$, for any strategy profile $\left(S_{i}, S_{-i}\right) \in \mathcal{S}^{m}$, such that $S_{i}=X_{0}$, and $S_{j} \subset X_{r}, \forall j \in \mathcal{M} \backslash\{i\}$, player $i$ colors initially $X_{0}$, then $X_{1} \cup \ldots \cup X_{m-1}$ and afterwards $X_{r} \backslash \bigcup_{j \in \mathcal{M} \backslash\{i\}} S_{j}$. Thereby, the whole network is eventually colored, and a maximal social welfare is obtained.

Also, the lowest social welfare is obtained for the PNE where the firm associated with the best quality product, according to $R^{\prec}$, picks the area $X_{0}$ as its strategy, and the remaining $m-1$ players pick the areas $X_{1}, \ldots, X_{m-1}$. Moreover, this lowest value is $m k$, and as a result the price of anarchy for the involved game is $|V| /(m k)$.

On the other hand, the highest social welfare is also obtained for the equilibrium where the firm associated with the best quality product, according to $R^{\prec}$, picks $X_{0}$, and the remaining $m-1$ players pick any of the mutual exclusive subsets of $X_{r}$. This best value is $|V|$, and as a result the price of stability for the involved game is 1 .

The previous facts complete our proof.

Case 2: The proof is similar to the proof of Theorem 9i, but over the right social network of Figure 4.

Proof of Theorem 9ii. In the first place, we refer the reader to Appendix F.1 for a discussion on the underlying structure of the involved social networks in Theorem 9ii. Also, recall Theorem 8i.

For the proof, we first assume that a unique node $v \in V$ exist such that $d_{v}^{\text {out }} \geq 1$, and we further assume that the maximal social welfare is obtained when the nodes $v, u$, where $u \neq v$, are initiated. We have the following cases: 
i. $(v, u) \notin E$. Evidently, the strategy profile $\left(S_{v}, S_{u}\right)$ constitutes a PNE. However, another PNE may exists where Player 1 initiates again node $v$, and Player 2 initiates a node $u^{\prime}$, such that $\left(v, u^{\prime}\right) \in E$. Therefore, the price of anarc hy is at most $S W(\mathbf{s}) /(S W(\mathbf{s})-1)$, where $\mathbf{s}=\left(S_{v}, S_{u}\right)$ is the social optimum.

ii. $(v, u) \in E$. Evidently, the strategy profile $\left(S_{v}, S_{u}\right)$ constitutes a PNE, and the price of anarchy for this case is one.

Next, we assume that at least two nodes $v, u \in V$ exists such that $d_{v}^{\text {out }}, d_{v}^{\text {out }} \geq 1$ and we further assume that the maximal social welfare is obtained when the nodes $v, u$ are initiated. We have the following cases:

i. $(v, u) \notin E$, and $(u, v) \notin E$. Assume that $d_{v}^{\text {out }} \geq d_{u}^{\text {out }}$ : Evidently, the strategy profile $\left(S_{v}, S_{u}\right)$ constitutes a $\mathrm{PNE}$, and the price of anarchy for this case is one.

ii. $(v, u) \in E$, and $(u, v) \notin E$. Evidently, the strategy profile $\left(S_{v}, S_{u}\right)$ constitutes a PNE, and the price of anarchy for this case is one.

iii. $(v, u) \notin E$, and $(u, v) \in E$. This case is symmetric to the previous one.

iv. $(v, u) \in E$, and $(u, v) \in E$. The strategy profile $\left(S_{v}, S_{u}\right)$ cannot be a PNE, therefore, either the maximal social welfare can achieved for another pair of nodes that correspond to one of the previous cases, or the game do not have any PNE, and as a result, we cannot study its price of anarchy. 